\title{
Laboratory experiments and simulations for solitary internal waves with trapped cores
}

\author{
Paolo Luzzatto-Fegiz ${ }^{1,2} \dagger$ and Karl R. Helfrich ${ }^{3}$ \\ ${ }^{1}$ Churchill College, University of Cambridge, Storey's Way, Cambridge CB3 0DS, UK \\ ${ }^{2}$ Department of Applied Mathematics and Theoretical Physics, University of Cambridge, \\ Wilberforce Road, Cambridge CB3 0WA, UK \\ ${ }^{3}$ Department of Physical Oceanography, Woods Hole Oceanographic Institution, \\ 266 Woods Hole Road Woods Hole, MA 02543-1050, USA
}

(Received ?; revised ?; accepted ?. - To be entered by editorial office)

We perform simultaneous, co-planar measurements of velocity and density in solitary internal waves with trapped cores, as well as viscous numerical simulations. Our setup comprises a thin stratified layer (approximately $15 \%$ of the overall fluid depth) overlaying a deep, homogeneous layer. We consider waves propagating near a free surface, as well as near a rigid no-slip lid. In the free-surface case, all trapped-core waves exhibit a strong shear instability. We propose that Marangoni effects are responsible for this instability, and use our velocity measurements to perform quantitative calculations supporting this hypothesis. These surface-tension effects appear to be difficult to avoid at the experimental scale. By contrast, our experiments with a no-slip lid yield robust waves with large cores. In order to consider larger-amplitude waves, we complement our experiments with viscous numerical simulations, employing a longer, virtual tank. Where overlap exists, our experiments and simulations are in good agreement. In order to provide a robust definition of the trapped core, we propose bounding it as a Lagrangian coherent structure (instead of using a closed streamline, as has been done traditionally). This construction is less sensitive to small errors in the velocity field, and to small three-dimensional effects. In order to retain only flows near equilibrium, we introduce a steadiness criterion, based on the rate of change of the density in the core. We use this criterion to successfully select within our experiments and simulations a family of quasi-steady, robust flows, which exhibit good collapse in their properties. The core circulation is small (at most, around $10 \%$ of the baroclinic wave circulation). The core density is essentially uniform; the standard deviation of the density, in the core region, is less than $4 \%$ of the full density range. We also calculate the circulation, kinetic energy, and available potential energy of these waves. We find that these results are consistent with predictions from Dubreil-Jacotin-Long theory for waves with a uniform-density, irrotational core, except for an offset, which we suggest is associated with viscous effects. Finally, by computing Richardson number fields, and performing a temporal stability analysis based on the Taylor-Goldstein equation, we show that our results are consistent with empirical stability criteria in the literature.

\section{Introduction}

Solitary internal waves are common in the ocean and atmosphere (e.g. Lien et al. 2012; Rao et al. 2004). Under appropriate background stratification and shear, solitary internal waves can develop recirculating regions, known as "trapped cores"; in a two-dimensional

$\dagger$ P.Luzzatto-Fegiz@damtp.cam.ac.uk 
representation of these waves, such regions are associated with closed streamlines, when observed in a reference frame moving with the phase velocity (e.g. King et al. 2011). In this paper our focus is on "mode-1" waves, which can propagate along a relatively steep stratification against a boundary or free surface (as opposed to the varicose-shaped "mode-2" waves, which can arise at a stratified layer between two essentially homogeneous, deep regions; e.g. Salloum et al. 2012). Trapped-core waves of the mode-1 variety often form in the coastal oceans, for example, when regular solitary waves (which may also form in deep water; Akylas et al. 2007, and references therein) break and rearrange through interaction with the continental shelf (Lamb 2002; Lien et al. 2012). Depending on the background stratification, waves propagating near the surface or near the bottom are possible (e.g. Scotti \& Pineda 2004; Moum et al. 2007b). It has been proposed that trapped cores may constitute an important vehicle for transporting mass, including biologically-active matter such as larvae and plankton (Scotti \& Pineda 2004). In addition, near-bottom waves may provide a significant source of sediment transport, and might carry sewage outflows back towards the near-shore region (Diamessis \& Redekopp 2006). These waves can also exert large forces on underwater structures, and their consideration is important in coastal engineering. In the atmosphere, trapped cores may be formed near the ground in the presence of a thermal inversion, with the wave typically initiated by a thunderstorm outflow (Doviak \& Christie 1989) or by local winds (an iconic example is provided by the Morning Glory cloud in western Australia; Clarke et al. 1981).

To model trapped-core waves, numerical solutions of the Dubreil-Jacotin-Long (DJL) equation are traditionally used (Dubreil-Jacotin 1934; Long 1953). The DJL equation is derived, in essence, by integrating the steady vorticity equation along a streamline that connects a point in the interior of the wave to an upstream location, which is at a known, background condition. However, the core constitutes a region whose streamlines are closed, and are not connected with the upstream flow. For this reason, models comprising recirculating regions require assumptions about the density and vorticity structure in the core. Several different choices can be used to find steady solutions (Davis \& Acrivos 1967; Derzho \& Grimshaw 1997; Helfrich \& White 2010; King et al. 2011).

Remarkably, inviscid simulations have shown that several radically different core properties can give robust waves. Lamb (2002) found cores with nonuniform density (more specifically, having a heavy core), and with circulation of sign opposite to the wave's baroclinic vorticity (which, in our study, is defined as positive; see e.g. figure 4 further below). Helfrich \& White (2010) found that sufficiently long computational runs eventually yielded cores with uniform density and negligible circulation. King et al. (2011) found DJL solutions whose cores have strongly positive circulations; their inviscid simulations showed that these solutions are robust.

The choice of core density and circulation is of significant importance, as it impacts the dispersion curves for the resulting family of waves (Helfrich \& White 2010), as well as their stability properties (King et al. 2011). In a flow involving small but finite viscosity, it is reasonable to expect that, over a sufficiently long time, viscous effects may select a single set of core properties (in accordance with a baroclinic version of the Batchelor theorem; see Batchelor 1956; Grimshaw 1969). Several field observations have yielded descriptions of core properties (e.g. Lien et al. 2012); however, it is difficult to establish whether these waves have indeed reached a quasi-steady state. To the best of our knowledge, no experimental studies to date have reported any core properties for mode- 1 waves (including volume, density, and circulation).

Grue et al. (2000), in the context of a broader study involving waves without trapped cores, reported the occurrence of a recirculating region in a few of their experiments. Carr et al. (2008), in a seminal work, performed an experimental study of instability in 
solitary internal waves of large amplitude. They considered waves propagating near a free surface, as well as near a no-slip boundary. They found that all of their trapped-core waves near a free-surface were unstable; furthermore, many of the waves near a no-slip boundary also showed instability. They proposed that free-surface deformations may be responsible for these and other differences between the two cases. These results of Carr et al. (2008) raise the deeper issue of whether a robust steady state may even be possible, for solitary internal waves with trapped core of moderate amplitude, in an experimental context. Carr et al. (2008) reported measurements of phase velocity and amplitude; to the best of our knowledge, other wave properties (such as energy) remain to be measured experimentally, for trapped-core waves.

We consider here also two further outstanding questions. The first involves the accuracy with which a DJL model can capture global properties of the waves, such as kinetic energy, and available potential energy. The second question involves testing commonly used empirical stability criteria for long internal waves (Troy \& Koseff 2005; Fructus et al. 2009), and establishing whether they accurately predict the onset of instability for trapped-core waves.

Summarising the outstanding points discussed above, this paper aims to address the following questions, for experimentally realized waves:

(a) Do trapped-core waves eventually settle to a family of quasi-equilibria? If so:

(b) What is the core circulation?

(c) What is the core density structure?

(d) What is the kinetic and available potential energy of trapped-core waves?

(e) Are commonly used empirical stability criteria also valid for trapped-core waves?

The rest of the paper is organized as follows. The experimental setup is briefly described in $\S 2.1$. We initially performed experiments involving waves propagating near a free surface; these results are reported in $\S 3$. We also examined waves propagating near a no-slip boundary; this data is described in $\S 4$. We found it valuable to complement our experimental observations with numerical simulations, as reported in $\S 2.2$. The combined data set is discussed in $\S 4.2$. The stability properties of these waves are assessed in $\S 6$. Conclusions follow in $\S 7$. To ease discussion of these flows, we propose referring to a "solitary internal wave with a trapped core" simply as a "solicore" (in this choice, we were of course inspired by the commonly used contraction "solibore").

\section{Experimental and computational setup}

\subsection{Experimental apparatus and procedure}

The experiments were performed using the solitary internal wave tank in the Geophysical Fluid Dynamics Laboratory at the Woods Hole Oceanographic Institution. This facility is described in detail in a separate forthcoming paper; here we provide a brief overview of its key characteristics.

The overall tank length, width and height are $8 \mathrm{~m} \times 0.4 \mathrm{~m} \times 0.5 \mathrm{~m}$, respectively. In all experiments, a deep and uniform layer of saltwater was covered with a shallow stratified layer of height $h$, yielding an overall depth $H$. An approximately linear stratification was produced using the two-bucket method (e.g. Economidou \& Hunt 2008), using mixtures of freshwater and filtered seawater. The background profile was measured using a conductivity-temperature probe (from Precision Measurement Engineering, see Head 1983); density was calculated using the Seawater library, version 3.3. A typical normal- 


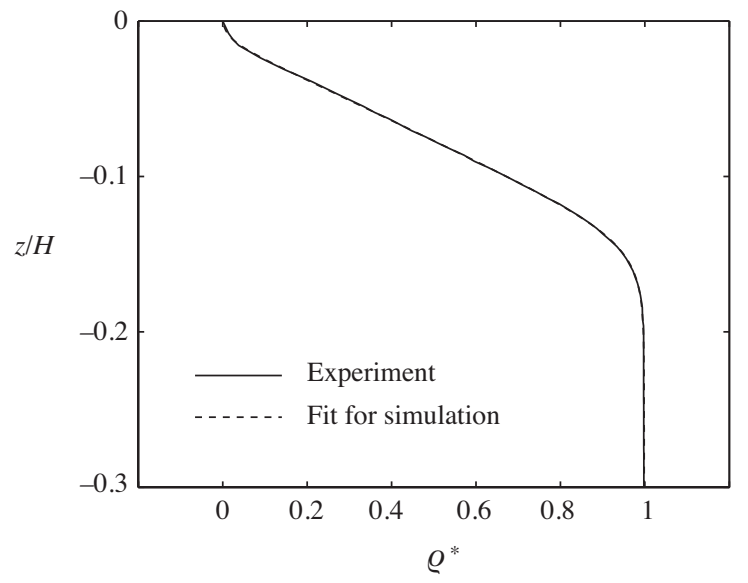

Figure 1. Typical normalized experimental background density profile (continuous line), and best-fit used for initializing the simulations shown later in $\S 2.2$ (dashed line). The two curves are nearly indistinguishable.

ized density profile is shown in figure 1 . The density $\rho$ is normalized using the densities at the top and bottom of the profile (i.e. $\left.\rho_{\text {top }}, \rho_{\text {bot }}\right)$, such that $\rho^{*}=\left(\rho-\rho_{\text {top }}\right) /\left(\rho_{\text {bot }}-\rho_{\text {top }}\right)$.

In all experiments reported here, $h / H \simeq 0.15$. In nearly all experiments, the total depth $H \simeq 0.35 \mathrm{~m}$; a few experiments (yielding the largest normalized-amplitude wave in $\S 4$ ) were performed with $H \simeq 0.14 \mathrm{~m}$. The typical internal buoyancy frequency, in the stratified layer, is $N_{0} \simeq 1.5 \mathrm{~s}^{-1}$. While experimental Reynolds numbers in the literature are often based on overall depth $H$, for small $h / H$ the most important length scale is arguably $h$. For a wave with phase velocity $c$, we therefore define a Reynolds number $R e_{h}=c h / \nu$, where $\nu$ is the dynamic viscosity. In our experiments, $R e_{h} \simeq 4,000$.

Waves are produced using a classic lock-release arrangement (e.g. Grue et al. 2000; Sutherland et al. 2013) at one end of the tank. Once the background stratification has been set up, a gate is introduced (approximately $0.3 \mathrm{~m}$ from one end of the tank), and additional liquid (closely matching the surface density) is added. Removing the gate generates a surface-propagating gravity current, whose head radiates long internal waves.

We image two downstream regions, located approximately $3.2 \mathrm{~m}$ and $6.2 \mathrm{~m}$ from the end of the tank. We acquire velocity data in a single plane by means of a particle image velocimetry (PIV) setup, comprising a Nd:Yag pulsed laser and CCD cameras with resolution $2048 \times 2048$ (LaVision). The field of view is approximately $0.35 \mathrm{~m}$ wide, and is located in a plane that is about $0.07 \mathrm{~m}$ away from the side of the tank, to minimize end effects while avoiding unacceptable optical distortion. The flow is seeded with $15 \mu \mathrm{m}$ Vestosint particles; a relatively high seeding density is used, thus enabling calculation of independent velocity vectors over a length scale of about $3 \times 10^{-3} \mathrm{~m}$. The typical data acquisition rate is $7 \mathrm{~Hz}$; velocity fields are calculated using DaVis 7.2.

To also measure density fields that are co-planar with the velocity data, we developed a planar laser-induced-fluorescence technique that makes possible the acquisition of accurate scalar concentration data even while using pulsed lasers (which are well-known to exhibit large fluctuations in power output; e.g. Melton \& Lipp 2003) and in the presence of PIV particles (which can scatter light, and thereby change local dye illumination and fluorescence; e.g. Law \& Wang 2000). The details of this technique are reported in a forthcoming paper. This methodology has enabled us to perform the first measurements of core density and Richardson number in these flows, as reported below in $\S 4$.

We perform experiments with a free surface, as well as with a no-slip boundary at 
the top. The latter is introduced shortly after the tank has been filled, using styrofoam panels whose width matches precisely that of the tank. We were careful to ensure that the panels had no seams or joins at least $1 \mathrm{~m}$ upstream and downstream of each imaging window, and covered the entirety of the tank, without appreciable gaps. A small opening was cut for the conductivity-temperature probe. The panels were lowered carefully until fully wetted, and were held in place rigidly.

Once a set of images is acquired, and the laboratory-frame velocity $\boldsymbol{u}=(u, w)$ and density $\rho$ are calculated, the phase velocity $c$ is measured by tracking the $x$-location of the wave trough. To achieve this, we follow the horizontal motion, at fixed depth, of the point at which $w\left(x, z=z_{0}\right)=0$. Due to the ease and relative accuracy with which $c$ can be found, we choose to plot all data as a function of phase velocity, in this paper (for the experiments with a no-slip lid, the uncertainty in $c$ is typically less than 1\%). The amplitude $\eta_{\text {pycno }}$ is defined as the displacement of the isopycnal at the lower end of the stratification, and is measured either by first computing streamlines in the moving frame (for small-amplitude waves, without instabilities or trapped cores) or by tracing the appropriate isopycnal in the density field.

To compute global wave properties (such as kinetic energy), we construct composite views of the waves, by shifting successive images horizontally by a distance $c \Delta t$ (where $\Delta t$ is the time between images). We calculated the Boussinesq kinetic energy $T$ and available potential energy $V$, per unit span, as:

$$
\begin{aligned}
T & =\frac{\rho_{\text {bot }}}{2} \int|\boldsymbol{u}|^{2} d A \\
V & =g \int\left[\int_{\bar{\rho}(z)}^{\rho}(z-\hat{z}(s)) d s\right] d A
\end{aligned}
$$

where $g$ is the acceleration due to gravity, $\rho$ is the wave density, $\bar{\rho}$ denotes the background profile, and $\hat{z}(\rho)$ is the inverse of $\bar{\rho}(z)$ (for a definition of the available potential energy, see e.g. Lamb \& Nguyen 2009).

We found it convenient to separate positive and negative contributions to the circulation, as this enabled distinguishing between circulation associated essentially with baroclinic vorticity $\left(\Gamma_{+}\right)$and circulation associated with the boundary layer against a no-slip boundary $\left(\Gamma_{-}\right)$:

$$
\begin{aligned}
& \Gamma_{+}=\int_{\zeta>0} \zeta d A \\
& \Gamma_{-}=\int_{\zeta<0} \zeta d A,
\end{aligned}
$$

where the vorticity is $\zeta=\partial u / \partial z-\partial w / \partial x$. We found it impractical to image the complete depth $H$, and only recorded data over a vertical distance $h_{\text {view }}$ (typically around $4 h$ ); below this depth, there is of course no change in density and vorticity, but there is nonnegligible motion. This does not affect $V$ and $\Gamma$ (which can be accurately calculated by taking integrals only over $-h_{\text {view }}<z<0$. However, $T$ must be computed by first separating the integral

$$
\frac{2}{\rho_{\text {bot }}} T=\int_{z>-h_{\text {view }}}|\boldsymbol{u}|^{2} d A+\int_{z<-h_{\text {view }}}|\boldsymbol{u}|^{2} d A
$$

For $z<-h_{\text {view }}$, the flow is essentially two-dimensional (even for turbulent waves). Introducing a streamfunction $\psi$ such that $(u, w)=(\partial \psi / \partial z,-\partial \psi / \partial x)$, and $\nabla^{2} \psi=\zeta$, 
performing integration by parts, and using the divergence theorem:

$$
\frac{2}{\rho_{\mathrm{bot}}} T_{z<-h_{\mathrm{view}}}=\int|\nabla \psi|^{2}=\oint \psi \nabla \psi \cdot \boldsymbol{n} d \ell+\int \zeta \psi d A,
$$

where $d \ell$ is an element of contour length, $\boldsymbol{n}$ is the unit outward-pointing normal to the contour, and we have omitted the integration bounds in order to ease readability.

The only region with nonzero $\zeta$ is the bottom boundary layer. Without loss of generality, we define $\psi$ such that $\psi=0$ on the boundary, and the area integral is assumed to be negligible. This also causes the bottom segment of the contour integral to vanish. Since the vertical ends of the contour also make no contribution (as $\nabla \psi$ is negligible for large $|x / h|)$, we have

$$
T_{z<-h_{\mathrm{view}}}=-\frac{\rho_{\mathrm{bot}}}{2} \int_{-X}^{X}(\psi u)_{z=-h_{\mathrm{view}}} d x,
$$

where $X \gg h$. Hence $T$ can be fully evaluated using only information from the $z>-h_{\text {view }}$ region.

The procedure to measure the properties of the core geometry is described in section $\S 4.2$. To help nondimensionalize the results, we calculate the linear long-wave phase velocity $c_{0}$ by solving the linearised DJL equation, using the experimentally measured background $N^{2}$ (a discussion of the linearised DJL equation can be found, for example, in King et al. 2011).

Unless otherwise noted, all remaining quantities are nondimensionalised by using $c_{0}$, $h$, and $\rho_{\text {bot }}$ as the velocity, length, and density scales, to yield the starred variables $T^{*}, V^{*}, \Gamma^{*}$, and so on (note that $\rho^{*}$ is an exception, and is defined earlier in this section).

\subsection{Numerical simulations}

Two-dimensional simulations were performed using the IAMR package, which consists of an algorithm for modeling incompressible viscous flows (Almgren et al. 1998). The tank length used was at least $24 H$ (tanks as long as $48 H$ were used for larger waves). No-slip conditions were used on the tank boundaries. The diffusivity, for the scalar equation modeling the density, is set to zero. While the code can employ an adaptive mesh refinement procedure, all runs were performed with a uniform cartesian mesh. Most simulations were two-dimensional, and employed a mesh size $\Delta x=\Delta z=H / 256$; runs at twice the resolution showed no appreciable differences in the results.

Selected runs were repeated in three dimensions, with periodic boundary conditions and an extent of $H / 4 \simeq 1.7 \mathrm{~h}$ in the spanwise direction. These three-dimensional runs exhibited a different initial stage, but eventually settled to two-dimensional quasi-steady states that were indistinguishable from those obtained from two-dimensional simulations. For convenience, we encoded a typical nondimensionalised experimental density profile by means of the function:

$$
\begin{gathered}
\hat{\rho}^{*}=\frac{s(z)-s(1)}{1-s(1)} \\
s(z)=1-\frac{a_{1}+a_{3}}{2} z-\frac{a_{2}-a_{1}}{2 \lambda_{1}} \ln \frac{\cosh \left(\lambda_{1}\left(z-z_{1}\right)\right)}{\cosh \left(\lambda_{1} z_{1}\right)} \\
-\frac{\left(a_{3}-a_{2}\right)}{2 \lambda_{2}} \ln \frac{\cosh \left(\lambda_{2}\left(z-z_{2}\right)\right)}{\cosh \left(\lambda_{2} z_{2}\right)}
\end{gathered}
$$

where the parameters are $a_{1}=-0.0215, a_{2}=-59.0, a_{3}=-0.00185, \lambda_{1}=35.0$, 


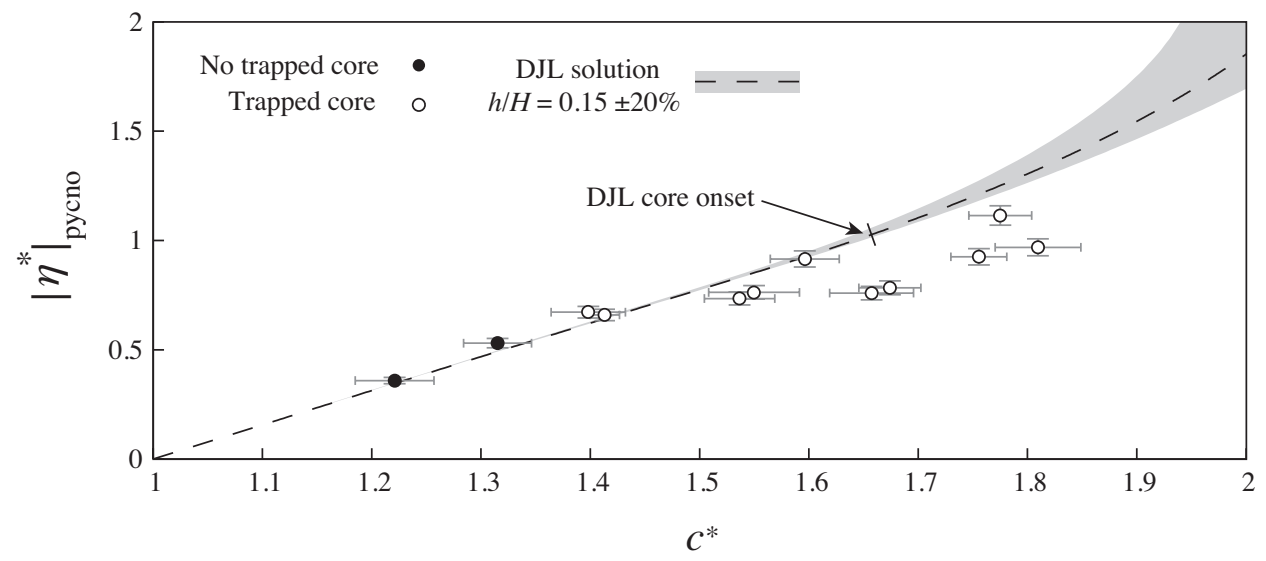

FIGURE 2. Normalized amplitude versus phase velocity for waves propagating near a free surface. Waves with and without trapped cores are denoted by open and closed symbols, respectively. A range of DJL solutions is shown for comparison.

$\lambda_{2}=101, z_{1}=0.859$, and $z_{2}=0.988$. The original and encoded profiles are shown by the continuous and dashed lines displayed earlier in figure 1 , and are nearly indistinguishable.

The results from the simulations were processed in essentially the same manner as those from the experiments, with the notable exception that it was not necessary to construct a composite view to compute integrals such as $T$ and $V$, and an instantaneous field was used instead (as for the experiments, care was taken in defining the $x$-extent for integration, to avoid including any following, smaller waves in the calculation).

\section{Solicores near a free surface}

At the beginning of our investigation, we performed a set of experiments with freesurface conditions; the resulting plot of phase velocity and amplitude is shown in figure 2 . For comparison, a family of DJL solutions with $h / H=0.15$ is shown by the dashed line. To provide an appreciation for the effect of changes in $h / H$, in this and other figures, the region spanned by DJL solutions with $h / H=0.15 \pm 20 \%$ has been shaded. These DJL solutions have irrotational, uniform-density cores.

It is immediately apparent that, while our measurements are never very distant from the DJL curve, there is appreciable scatter in the data. Another remarkable feature of these results is that trapped cores are first observed for $c^{*} \simeq 1.4$, well below the DJL value of $c^{*}=1.64$. Furthermore, while this is not apparent from this data set, all these solicores exhibit shear instabilities, as shown later in figure $3 a$. Our vorticity measurements, shown in figure $3 a$, show a strong shear layer forming ahead of the wave, and being subducted at the front of the core.

As briefly noted in $\S 1$, the effect of a free-surface boundary condition (as compared to a no-slip boundary) has been previously considered by Carr et al. (2008), who reported that free-surface conditions were more likely to yield instability, and produced smalleramplitude waves, for given phase velocity. Carr et al. proposed that surface deformation may play an essential role in this problem. They tested this hypothesis by introducing surfactants (thereby enabling greater surface deformations), which were found to lead to smaller amplitudes, and stronger instabilities, in their free-surface experiments. We initially sought to employ this hypothesis to explain the presence of this shear layer, 
but could not find a quantitatively viable mechanism that would produce shear through surface deformation.

To gain further insight into this problem, we constructed a large composite image, for a single experiment, as shown in figure $3 a$. This reveals that, remarkably, the shear layer extends forward at least up to $x \simeq 4 H \simeq 27 h$, thereby suggesting a phenomenon capable of long-range effects. Here we propose considering the tangential force balance at the surface, namely:

$$
\frac{\partial \gamma}{\partial x}=\mu \frac{\partial u}{\partial z}
$$

where $\mu$ is the dynamic viscosity, and $\gamma$ is the surface tension. This suggests that the wave may be producing a surface tension gradient, which, in turn, is capable of producing shear. At the front of the core, fluid converges at the surface (towards a stagnation point), before being transported below the core. It is therefore plausible that this would locally increase the concentration of any surfactant present on the surface, thus reducing the local value of the surface tension, and driving a Marangoni flow.

While we cannot directly measure surfactant concentration or surface tension in our experiment, we may test our hypothesis quantitatively as follows. We use equations describing conservation of momentum and conservation of surfactant to estimate, from our velocity measurements, the surface tension $\gamma$ and surfactant concentration $\phi$. We can then check whether these two quantities vary together in a manner that is consistent with the classical surfactant-surface tension relation provided by the appropriate Gibbs isotherm (Leal 2007).

To find the change in surface tension relative to the background value, we integrate (3.1):

$$
\gamma(x)-\gamma_{\infty}=-\mu \int_{x}^{\infty} \frac{\partial u}{\partial z} d x,
$$

where $\gamma_{\infty}$ denotes the background value of $\gamma$, ahead of the wave. The curve calculated from (3.2) is shown by the continuous line in figure $3 b$. To estimate the relative change in surfactant concentration at the surface, we assume that adsorption and desorption are negligible, such that the total amount of surfactant at the surface is conserved. Assuming also that the flow is steady in a frame of reference moving with the wave, and the motion along the spanwise direction is negligible at the surface:

$$
\frac{d[(u-c) \phi]}{d x}=0
$$

which can be integrated to give $\phi$ as a fraction of its background value $\phi_{\infty}$

$$
\frac{\phi(x)}{\phi_{\infty}}=\frac{c}{c-u},
$$

The value of $\phi / \phi_{\infty}$ calculated according to (3.4) is shown by the dashed line in figure $3(c)$. Sufficiently close to the wave, desorption may become significant, and the flow will be three-dimensional. Therefore the region with $x / H<1.5$ has been grayed out in figures $3(b)$ and $(c)$.

The range of surface concentrations and surface tension variations calculated by (3.2) and (3.4) suggest that the relationship between $\gamma$ and $\phi$ should be accurately modelled by the linear approximation (Leal 2007):

$$
\gamma-\gamma_{\infty}=R T \phi_{\infty}\left(1-\frac{\phi}{\phi_{\infty}}\right),
$$

where $R$ is the universal gas constant, and $T$ is the absolute temperature. We use (3.5) to 


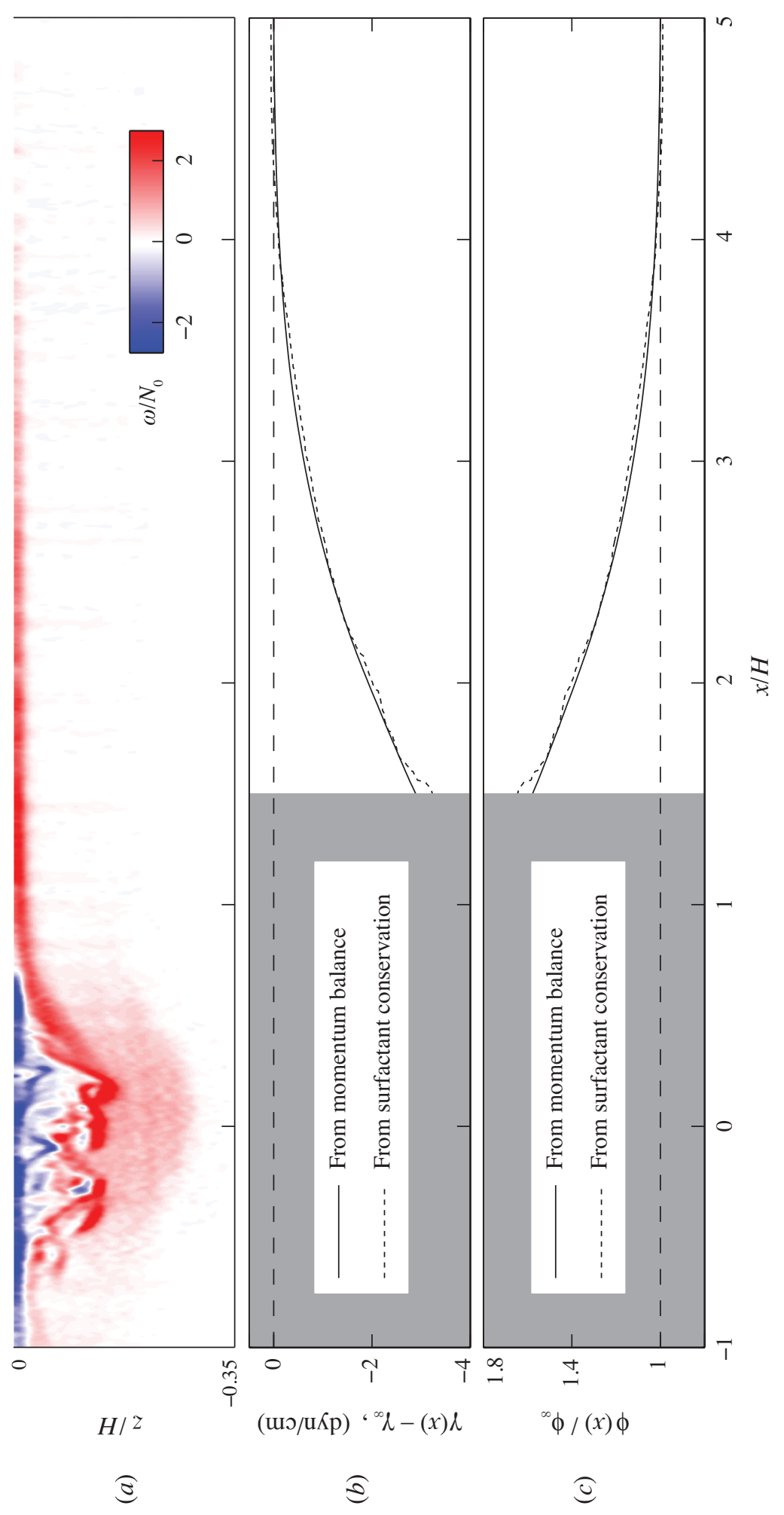

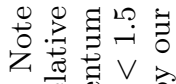

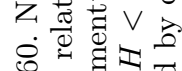

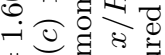

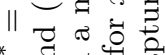

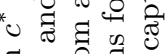

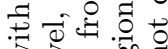
30 耐。 चै च उ $600 \dot{0}$

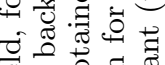
త 总密要焉点 焉范

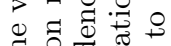

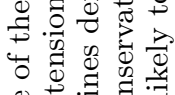
语 范 흘 द्व 唡.

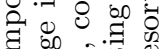
잉 क्ष ¿ $\approx$.

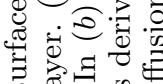

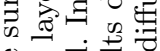
Ð $\approx \frac{0}{\omega} \stackrel{0}{0}$ ఠี Ð

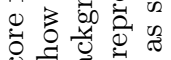

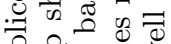
क 월 F⿻워 उ 중 \&

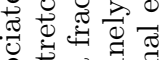
o

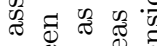

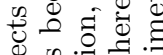

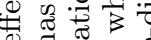
च. $\infty$ 政 䒕.

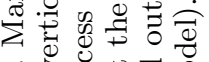

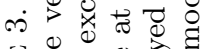
전

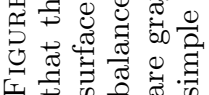


determine empirically $\phi_{\infty}$ from our calculated $\gamma-\gamma_{\infty}$ and $\phi / \phi_{\infty}$; a least-square procedure for $x / H>1.5$ yields $\phi_{\infty} \doteq 1.96 \times 10^{-6} \mathrm{~mol} \mathrm{~m}^{-2}$. In practical situations, for surfactants that are below the micellar concentration, $\phi_{\infty}$ is typically in the range $1-4 \times 10^{-6} \mathrm{~mol} \mathrm{~m}^{-2}$ (Rosen \& Kunjappu 2012). The fact that the value of $\phi_{\infty}$ estimated from our experiment falls within this range strongly suggests that surface tension gradients (driving a Marangoni flow) are indeed responsible for setting up the strong shear layer observed in free-surface experiments.

In oceanic contexts, Marangoni effects associated with internal waves have long been known to affect surface wave patterns (e.g. Kropfli et al. 1999). However, our finding is that, at the laboratory scale, Marangoni effects can also affect the interior of the fluid. The mechanism by which this occurs may be summarized as follows:

(a) The wave sets up a Marangoni flow, extending several wavelengths ahead;

(b) The Marangoni flow is associated with a strong surface shear layer;

(c) In interacting with the trapped core, the shear layer is subducted, becoming a free shear layer in the interior;

(d) This free shear layer exhibits a strong Kelvin-Helmholtz instability.

Of course, these Marangoni effects have a negligible impact on the interior of fullscale oceanic waves. Unfortunately, it is exceedingly difficult to perform experiments completely free of surfactants, without resorting to the use of a clean room and distilled water, which is not practically feasible in this case (for an example of the challenges associated with such experiments, in the context of studying rising bubbles, see Wu \& Gharib 2002). We therefore set aside free-surface experiments, and focus the rest of this paper on waves near a no-slip boundary.

\section{Solicores near a no-slip boundary}

\subsection{Experiments}

Introducing the rigid-lid setup described in $\S 2.1$, and running the experiments again, we are able to observe stable waves with trapped cores. Figure 4 shows the vorticity and density fields for three selected waves of progressively larger amplitude. The right-hand panels show sections of vorticity and density through the trough of each wave. In all of these flows, a strong boundary layer (corresponding to a region of negative vorticity) develops at the no-slip surface. However, this boundary layer remains attached to the surface, and does not give rise to a free shear layer (as was the case in the free-surface experiments).

Another small feature of these flows stands out. In all of our experiments, the baroclinic vorticity exhibited "bands"; these are visible both in the fields and in the vertical crosssections. We initially considered that these may be due to experimental errors. However, we found that, when differentiating the (seemingly smooth) background $\rho$, corresponding bumps appeared in the resulting $N^{2}$. We tested this link by using the measured $N^{2}$ to compute DJL solutions, which did indeed display similar vorticity bands. In retrospect, we found that such vorticity bands appear also in previous PIV measurements of long internal waves, although they appear to have been treated as PIV errors (e.g. figures 10-12 in Grue et al. 2000); nevertheless, it is not clear how one might avoid these imperfections when setting up the background stratification.

As explained in $\S 1$, a crucial goal of our investigation involves measuring core properties such as volume, circulation, and density structure. To achieve this, we need to first delimit the core boundary for each wave. While this has never been done from experimental data (to the best of our knowledge), oceanic and atmospheric observations 
$(a)$
$\ominus$
$\sum_{3}^{+1}$
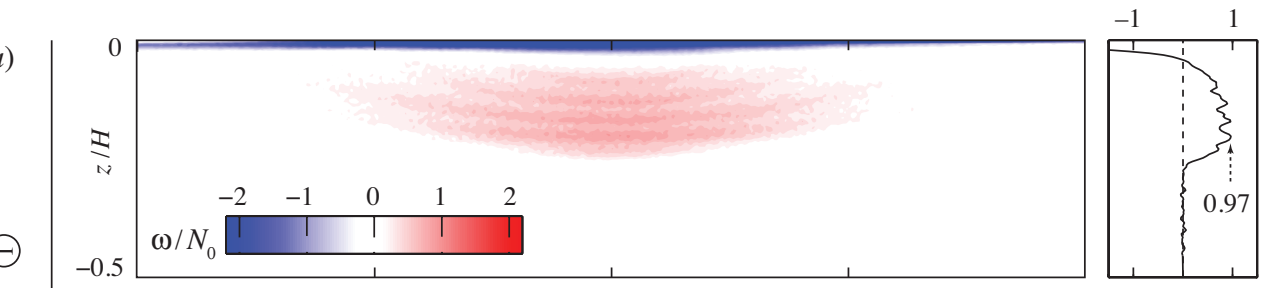

事
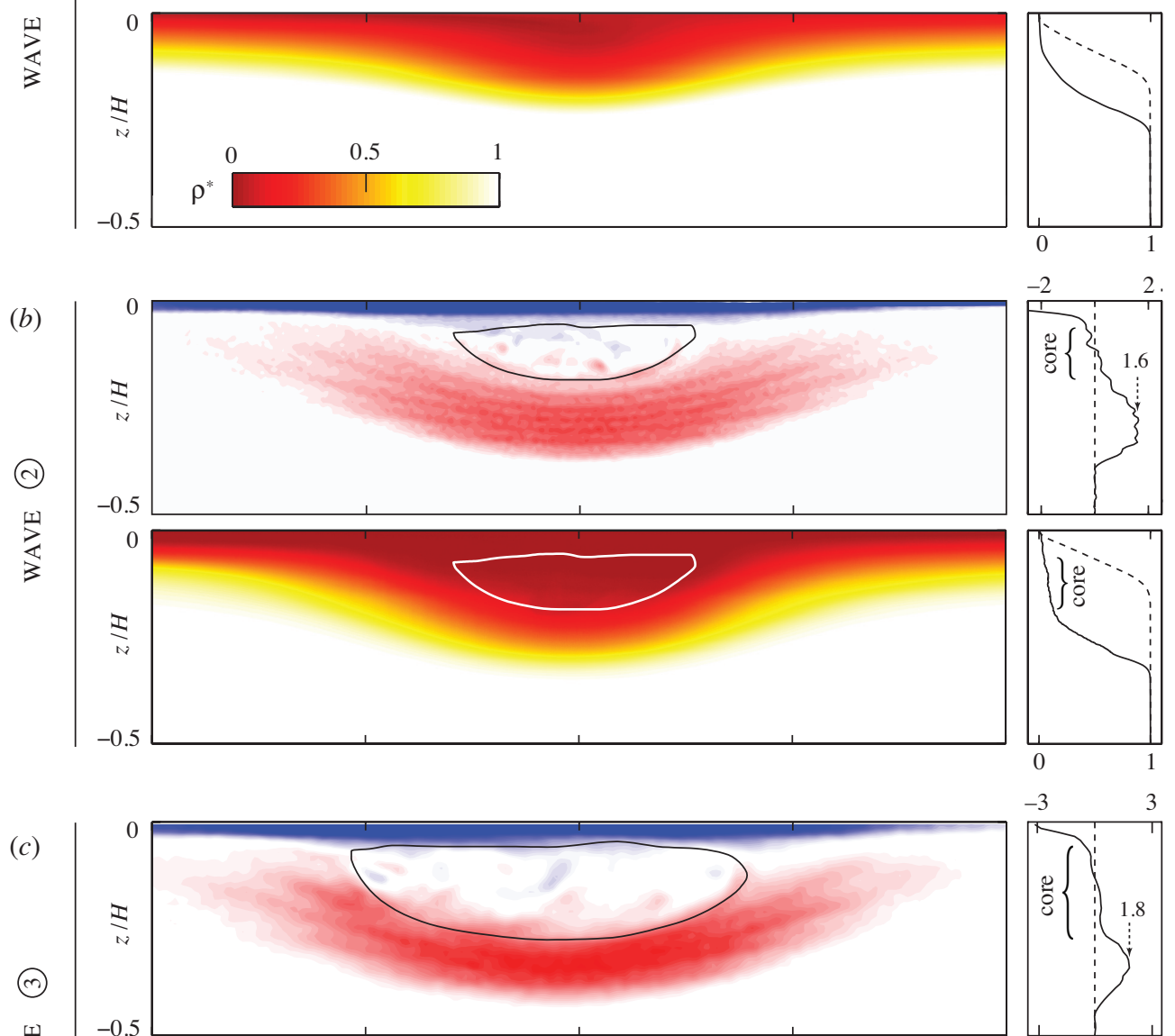

$\frac{1}{3}$

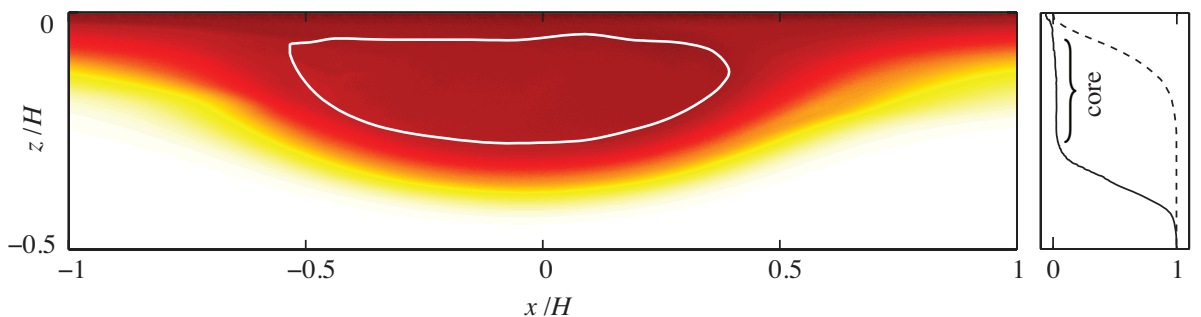

FiguRE 4. Sets of vorticity (top) and density (bottom) fields for three selected waves. In the left-hand panels $(b)$ and $(c)$, the core regions estimated by the LCS procedure are shown. The right-hand panels show vertical slices at each wave trough; the background conditions are marked by the dashed lines. The wave numbering corresponds to the amplitude plot in figure 5 . 
typically resort to using streamlines, in a frame of reference moving with the wave (e.g. Lien et al. 2012). However, we found that, in the wave interior, even relatively weak three-dimensional effects (of the order $\partial u / \partial x+\partial w / \partial z \sim 0.1 N_{0}$ ) were sufficient to create considerable uncertainties in the streamlines, and therefore in the core properties.

To compute a more robust definition of the core boundary, here we introduce to this problem the use of Lagrangian Coherent Structures (LCS). We employed the traditional definition, for an LCS, as the intersection between forward- and backward-time ridges of the Finite-Time Lyapunov Exponent (FTLE) field (for a general discussion of FTLEs, see e.g. Haller 2005; Shadden et al. 2006).

Examples of the core regions bounded by the LCS procedure are shown in figure 4(b) and $(c)$, by means of thin black lines that have been overlaid on the vorticity and density fields. Note that the core regions align well with the edges of the baroclinic vorticity in the waves; this lends further confidence to our use of an LCS procedure to identify trapped cores. The panels on the right-hand side of figure 4 show vertical slices of vorticity and density at the wave trough; the extent of the core is also marked in these plots. The interior of each core has relatively little vorticity (by comparison to the baroclinic vorticity below the core), and approximately uniform density. Our findings therefore support the use of models with uniform core density and zero core circulation, to model these waves.

Incidentally, we found that sometimes two trapped-core waves with similar $c^{*}$ exhibited different stability properties, as well as different core geometries. We hypothesize that these differences may be attributable mainly to transient effects associated with the details of the wave generation process, and the initial evolution. For example, we measured the fluid density behind the lock gate, and realized that much of this fluid was slightly less dense than the lightest fluid in the main portion of the tank; typically $\min \left(\rho_{\text {lock }}^{*}\right) \simeq-0.05$. In this particular circumstance, this density structure can be shown to be the result of the use of the two-bucket method. Once we had completed filling the background stratification, the valve connecting the two buckets was shut, thus fixing the value of the density in the "heavier fluid" bucket. However, since this bucket was connected to the wave tank by a finite length of hose, a small density mismatch was set up between the fluid at the end of the hose (and at the top of the tank stratification) and the fluid in the bucket. This meant that relatively light fluid from the lock could initially become entrained in the core, contributing to sharp density gradients, and therefore to instabilities that might be seen as spurious in our experimental setup (however, such entrainment mechanics can occur in natural settings; see e.g. Lamb \& Farmer 2011). It would be interesting to explore more efficient mechanisms for producing long internal waves, with shorter transient effects (for example, in the spirit of the "new wave" apparatus, for generating internal wave beams, developed by Mercier et al. 2010). However, this is beyond the scope of our present study.

In order to control for this and other transient effects, we introduce a measure of unsteadiness, for solicores, defined as:

$$
\left\langle\rho_{\tau}^{*}\right\rangle \equiv \int_{A_{\text {core }}^{*}}\left|\frac{\partial \rho^{*}}{\partial \tau^{*}}\right| d A^{*}
$$

where $\frac{\partial}{\partial \tau}=\frac{\partial}{\partial t}+c \frac{\partial}{\partial x}$ is the time derivative in the frame of reference moving with the wave. We found that, by restricting our results to cases meeting $\left\langle\rho_{\tau}^{*}\right\rangle<0.06$, we could retain robust waves for relatively large amplitudes, as shown by the circles in figure 5 . The scatter in wave properties was also considerably reduced, and became consistent with the estimated measurement uncertainties.

An adverse effect of conditionally sampling our results in this manner is that a smaller 


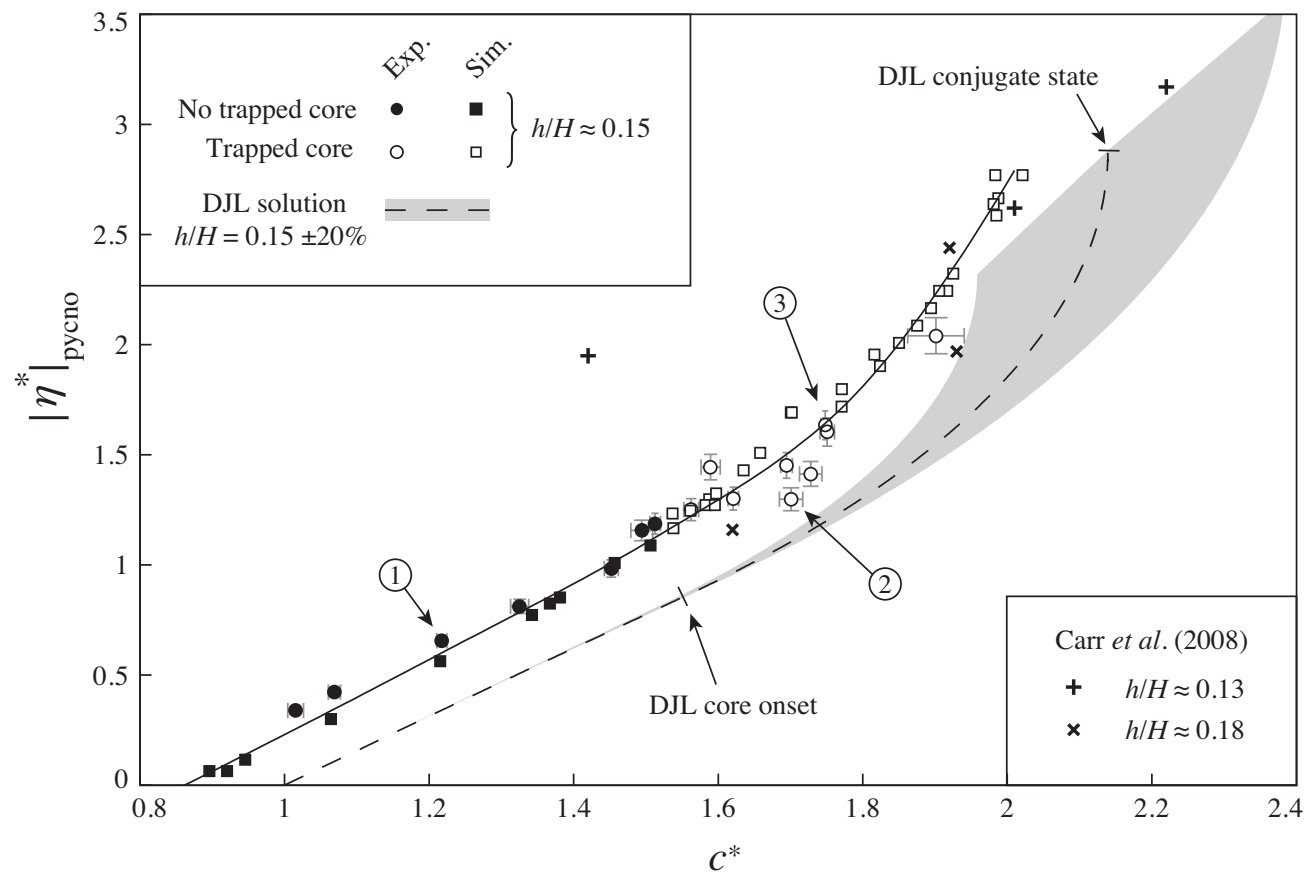

Figure 5. Pycnocline displacement versus phase velocity. Circles and squares denote experiments and simulations. Open and filled symbols represent waves with and without trapped cores. The corresponding stagnant-core DJL solution, up to the limiting (conjugate) state, is shown by the dashed line. For these results, $\left\langle\rho_{\tau}^{*}\right\rangle<0.06$. The experimental results of Carr et al. (2008) for $h / H \simeq 0.13$ and 0.18 are also shown using pluses $(+)$ and crosses $(\times)$.

data set is retained. In order to complement these results, and examine robust waves with very large amplitudes, we performed a set of viscous numerical simulations (information about the numerical setup is reported earlier in $\S 2.2$ ).

\subsection{The combined experimental and numerical data set}

As noted earlier, the velocity-amplitude plot is shown in figure 5, with circles and squares denoting our experimental and numerical results, respectively. Open and closed symbols correspond to waves with and without trapped cores. The overall trend is emphasized by the black continuous line fit to the data, whereas (following the same notation introduced earlier in figure 2) the DJL solution is shown by the dashed line. These DJL solutions were computed with a revised version of the code of Helfrich \& White (2010), which is unaffected by the minimum value of Richardson number in the flow (such that the code can also compute solutions that may be susceptible to shear instability, or other instabilities). This method enables the computation of waves approaching the limiting (conjugate) state.

Several significant observations can be made from this plot. Firstly, the experiments and simulations are in remarkably close agreement. This agreement includes the value of $c^{*}$ demarcating the emergence of a trapped core, which the combined dataset yields as $c_{\text {core }}^{*}=1.52 \pm 0.01$.

Secondly, for any value of $c^{*}$, the amplitude of our waves is larger than for the DJL solution; this is not explained by possible small differences in $h / H$ between theory and experiment/simulation, as these only have an appreciable effect at larger $c^{*}$. Instead, it seems plausible to suggest that the increase in amplitude may be due to viscous effects. 
More specifically, the viscous boundary layer in these waves (corresponding to the negative vorticity in figure 4) displaces the flow away from the boundary, therefore enhancing the downward displacement of the isopycnals. Measurements from experiments and simulations indicate that the boundary layer displacement thickness is indeed of the order $\delta / h \sim 0.2$, which is consistent with the observed gap between our values of $\eta_{\text {pycno }}^{*}$ and those from DJL theory (see figure 5).

Thirdly, we remark that it is possible to produce very small waves that are slower than the (inviscid) linear phase velocity, i.e. $c^{*}<1$, suggesting that at least part of the effect of a viscous boundary layer involves reducing the wave propagation velocity, relative to an inviscid wave.

Figure 5 also shows results from Carr et al. (2008) for $h / H \simeq 0.13$ and 0.18 , with a no-slip lid (they also performed measurements for $h / H \simeq 0.09$ and 0.27 , but these are not shown here, as results are sensitive to the value of $h / H$, making a direct comparison difficult). The experiments of Grue et al. (2000) focused on a fractional stratification depth of $h / H \simeq 0.24$; since our experiments involve waves with an $h / H$ that is approximately $60 \%$ smaller, the two data sets are not directly comparable. For the data of Carr et al. (2008), an offset from the DJL solution, similar to that displayed by our results, is also visible; this may be expected, as the ranges of Reynolds numbers in the two sets of experiments are very similar (it appears that Carr et al. 2008, did not discuss viscous effects in their work).

Key properties describing core geometries are reported in figure 6: panels $(a),(b)$ and (c) show the cross-sectional area $A_{\text {core }}$, height $h_{\text {core }}$, and length $L_{\text {core }}$ of the LCS region bounding the core (as shown earlier in figures $4 b$ and $c$ ). The collapse in these quantities is remarkable, given the relative difficulty associated with performing these measurements. Apart from a clear offset, these core measurements seem to parallel closely DJL theory. A feature worth mentioning here is that, shortly after the core first emerges at phase velocity $c^{*}=1.52$, this data suggests that $h_{\text {core }}$ and $L_{\text {core }}$ are well approximated by a fit of the type $\left(c^{*}-c_{\text {core }}^{*}\right)^{1 / 2}$, such that $A_{\text {core }}^{*} \propto\left(c^{*}-c_{\text {core }}^{*}\right)$, as indeed seems to be the case from figure $6(a)$. To the best of our knowledge, these results constitute the first laboratory measurements of trapped-core volume.

Note that, in figures $6(a)$ and $(c), A_{\text {core }}$ and $L_{\text {core }}$ appear to exhibit vertical asymptotes, suggesting that the limiting phase velocity (for this $h / H$ and $R e_{h}$ ) has essentially been reached. In other words, these results suggest that the full range of available $c^{*}$, for these experimental conditions, is spanned by our combined experimental and numerical data set.

We now come to addressing in a precise manner questions $(b)$ and $(c)$ that were raised in $\S 1$, namely, what are the core density and circulation? We previously showed density and vorticity fields, together with one-dimensional profiles at the wave troughs, which suggested that the core would have negligible circulation and density variation (see figure 4). Figure $7(a)$ shows the core circulation, plotted as a fraction of the positive (baroclinic) wave circulation $\Gamma_{+}$. No discernible trend seems to emerge, and $\left|\Gamma_{\text {core }} / \Gamma_{+}\right|$always seems to be less than about 0.1 . To help establish whether this small value of circulation should be accounted for in DJL models, here we also consider a DJL theory, which has a nominally irrotational core (this theory is described in detail in $\S 6$ of Helfrich \& White 2010). Instead of handling the core region as perfectly irrotational, this model lets $N^{2}$ reach a constant value through a rapid but smooth transition of the algebraic type. Therefore, even in such a nominally irrotational core model, a thin band of vorticity at the core edge can yield a nonzero $\Gamma_{\text {core }}$ (using an exponential transition, as done by King et al. 2011 , might be expected to reduce the value of $\left.\Gamma_{\text {core }}\right)$. This theoretical value of $\Gamma_{\text {core }}$ is of the same order as the one observed experimentally, suggesting that available DJL models 

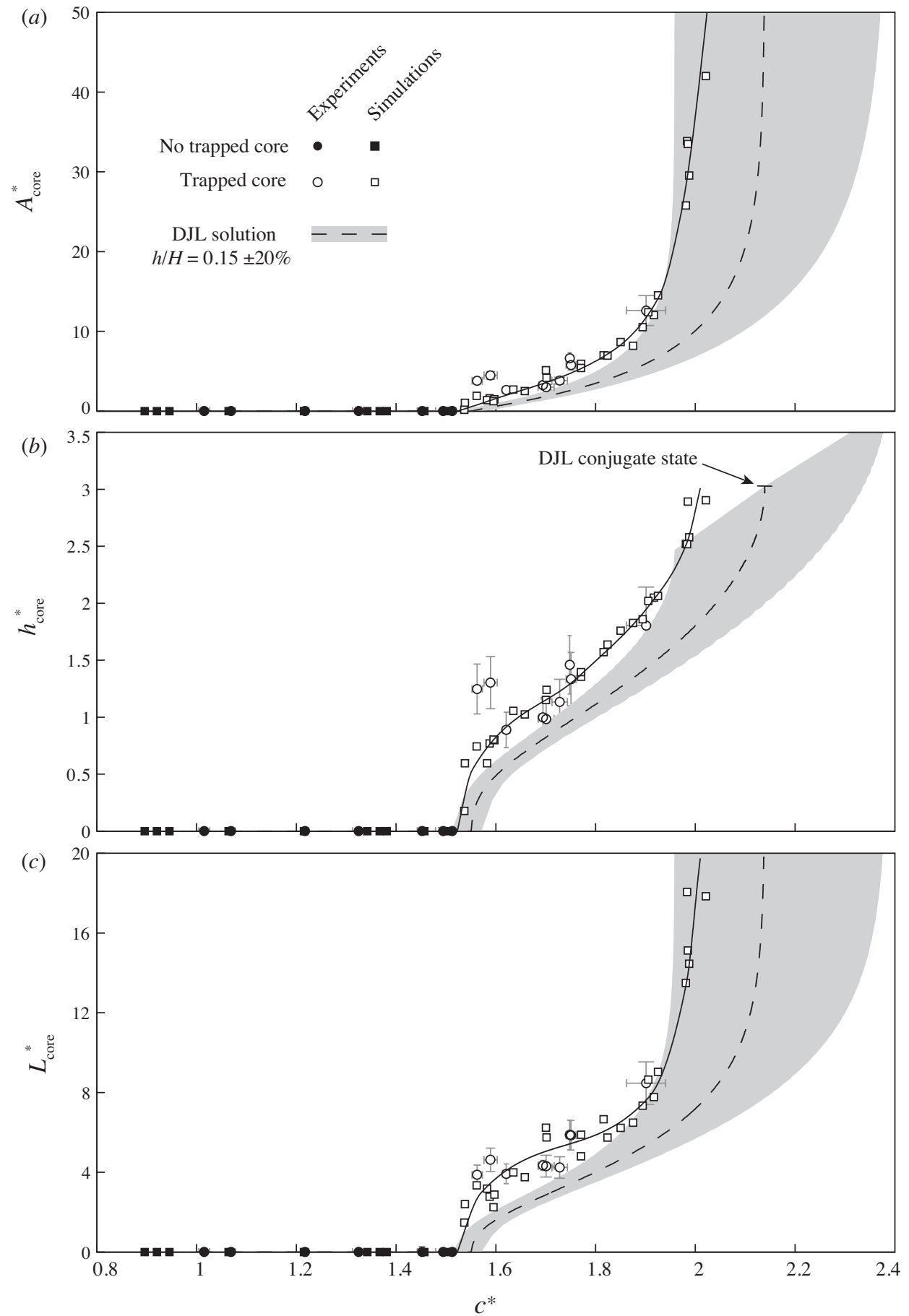

Figure 6. Properties describing core geometry. $(a)$ area, $(b)$ height and $(c)$ length. Symbols as in figure 5. 

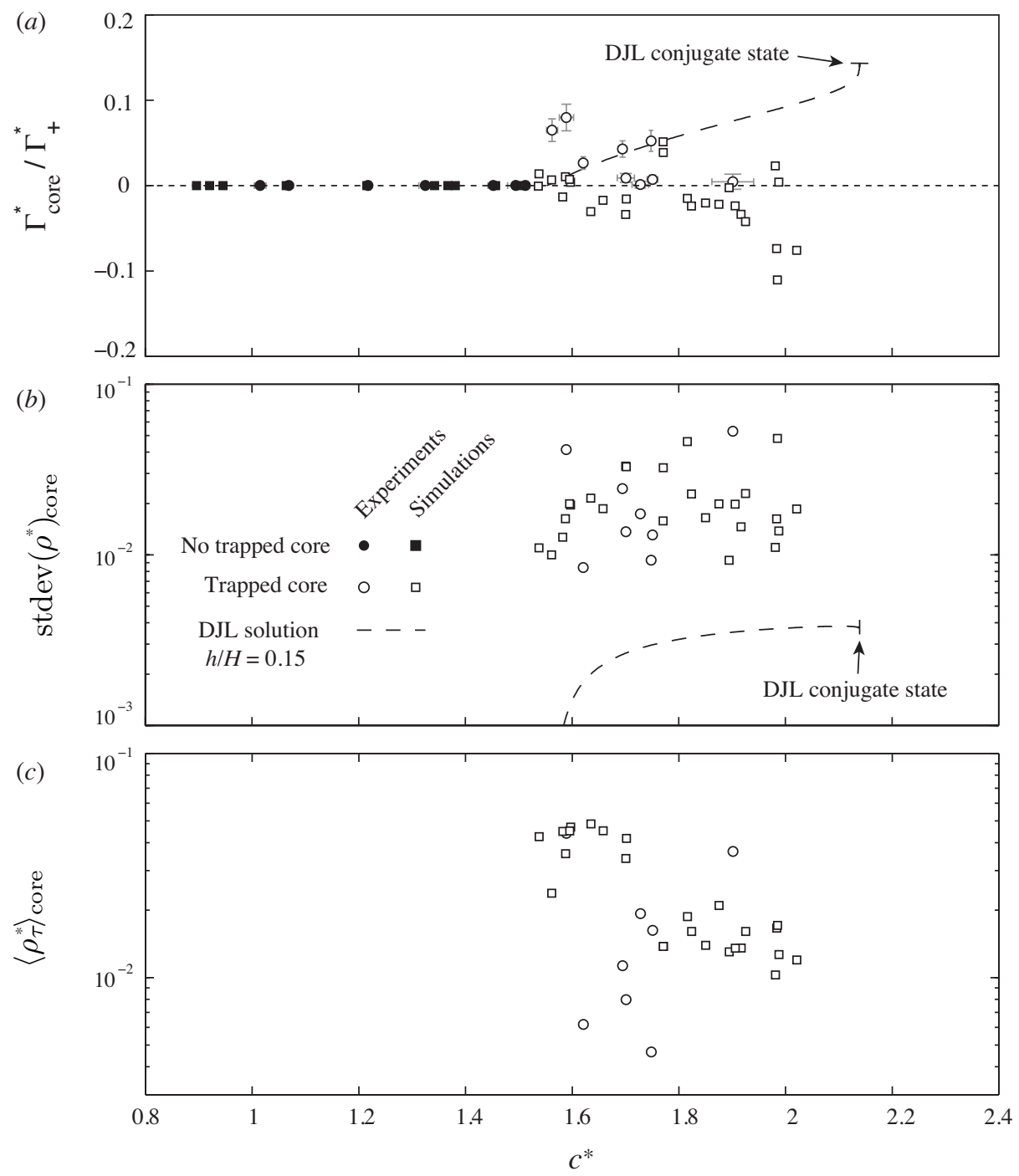

Figure 7. Additional core properties. (a) core circulation, expressed as a fraction of the baroclinic wave circulation. (b) density standard deviation in the core. (c) magnitude of the time derivative of the density (in a frame of reference moving with the wave), averaged over the core. This is a measure of wave steadiness. For comparison, properties for a DJL model with a nominally irrotational, uniform-density core are also shown. These results suggest that the uniform-density, irrotational core assumption constitutes a reasonable approximation.

(with nominally irrotational cores) are indeed suitable to describe these waves, and that this small value of core circulation is likely to be dynamically negligible.

To provide a quantitative measure of the core density structure, we computed the standard deviation of $\rho^{*}$ inside the core (i.e. $\operatorname{stdev}\left(\rho^{*}\right)_{\text {core }}$ ), as shown in figure $7(b)$. The largest value found is about $\operatorname{stdev}\left(\rho^{*}\right)_{\text {core }} \simeq 0.04$, which indicates that the core is, to a very good approximation, uniform. (For completeness, we also computed $\operatorname{stdev}\left(\rho^{*}\right)_{\text {core }}$ for a family of DJL solutions with a nominally irrotational core; this is shown by the dashed line in the figure.) For reference, panel (c) in figure 7 also shows the unsteadiness measure $\left\langle\rho_{\tau}^{*}\right\rangle$ for our waves. 


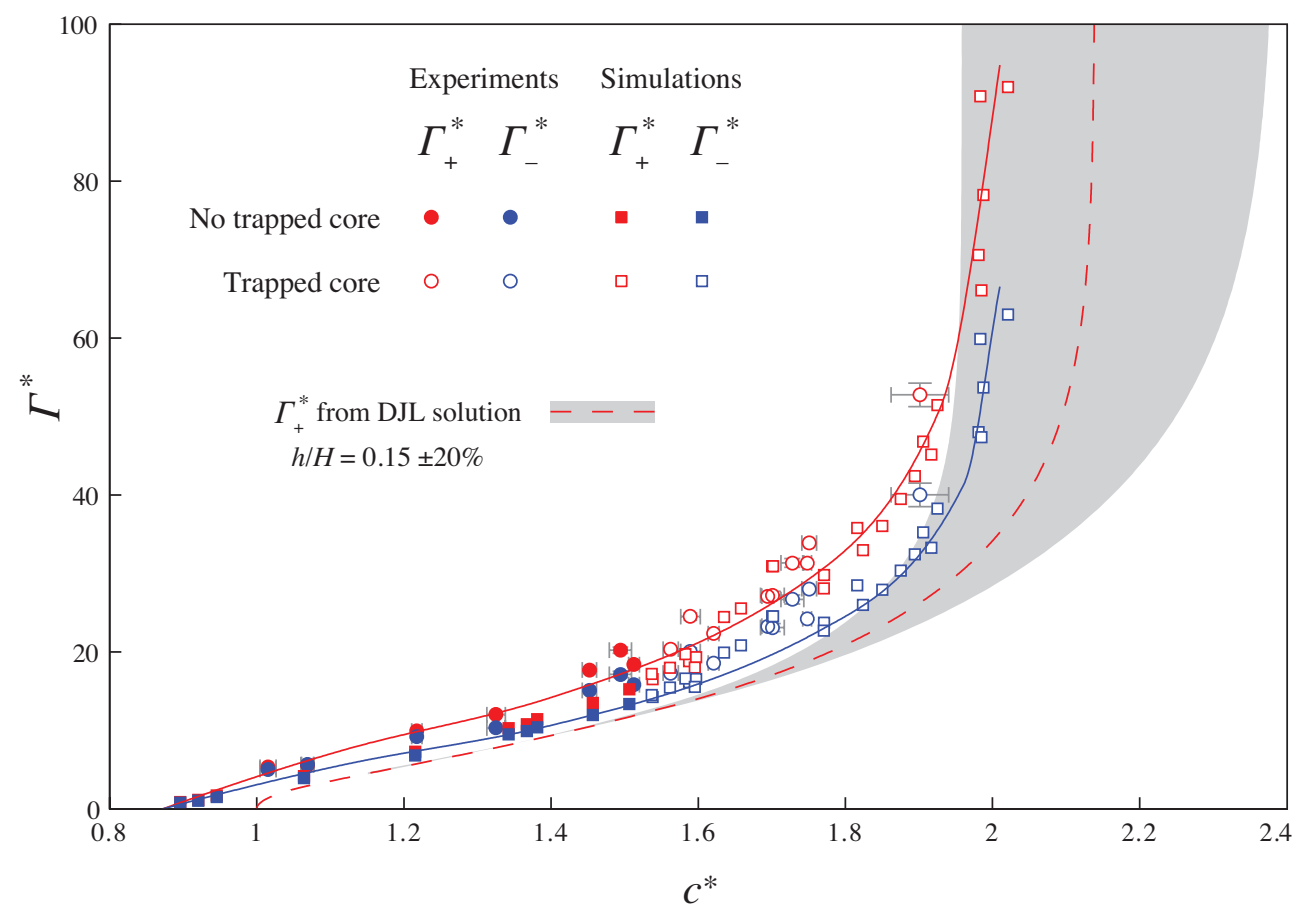

FiguRE 8. Circulation versus phase velocity. Red symbols correspond to positive circulation associated with baroclinic vorticity generation. Blue symbols represent negative circulation associated with the boundary layer. The remaining notation is the same as in figure 5 .

We next consider wave circulation, which is reported in figure 8 . The red symbols show the circulation associated with the baroclinically produced vorticity $\Gamma_{+}$(which has positive sign, and is displayed in red in the vorticity contour maps of figure 4). The corresponding value of the circulation from the DJL model is shown by the red dashed line (once again, with bounds for $h / H=0.15 \pm 20 \%$ shown in gray). The absolute value of the circulation $\Gamma_{-}$associated with the viscous boundary layer above the wave is shown by the blue symbols in figure 8 (of course, since the DJL model is inviscid, it does not yield a prediction for this quantity). Incidentally, note that the presence of no-slip conditions implies that the total circulation in the domain must be zero at all times. This fact allows one to use figure 8 to also calculate the circulation associated with the bottom boundary layer, simply as $\left|\Gamma_{-}\right|-\left|\Gamma_{+}\right|$.

Figure $9(a)$ and $(b)$ show the kinetic energy $T^{*}$ and available potential energy $V^{*}$. Figure $9(c)$ shows their ratio $T^{*} / V^{*}$. There is still some scatter in the experimental data, although this is consistent with the magnitude of the uncertainty shown in the figure. The simulations (after enforcing the steadiness requirement $\left\langle\rho_{\tau}^{*}\right\rangle<0.06$ ) collapse to form a distinct curve, which is emphasized by the black line. A key finding, from this plot, is that for low phase velocities, waves with $T^{*}<V^{*}$ are possible. Overall, the observed $T^{*} / V^{*}$ is slightly lower than predicted by DJL theory, and takes a maximum value of about 1.2 around $c^{*} \simeq 1.7$, whereas the maximum value of $T^{*} / V^{*}$ from experiments is approximately 1.5 . 

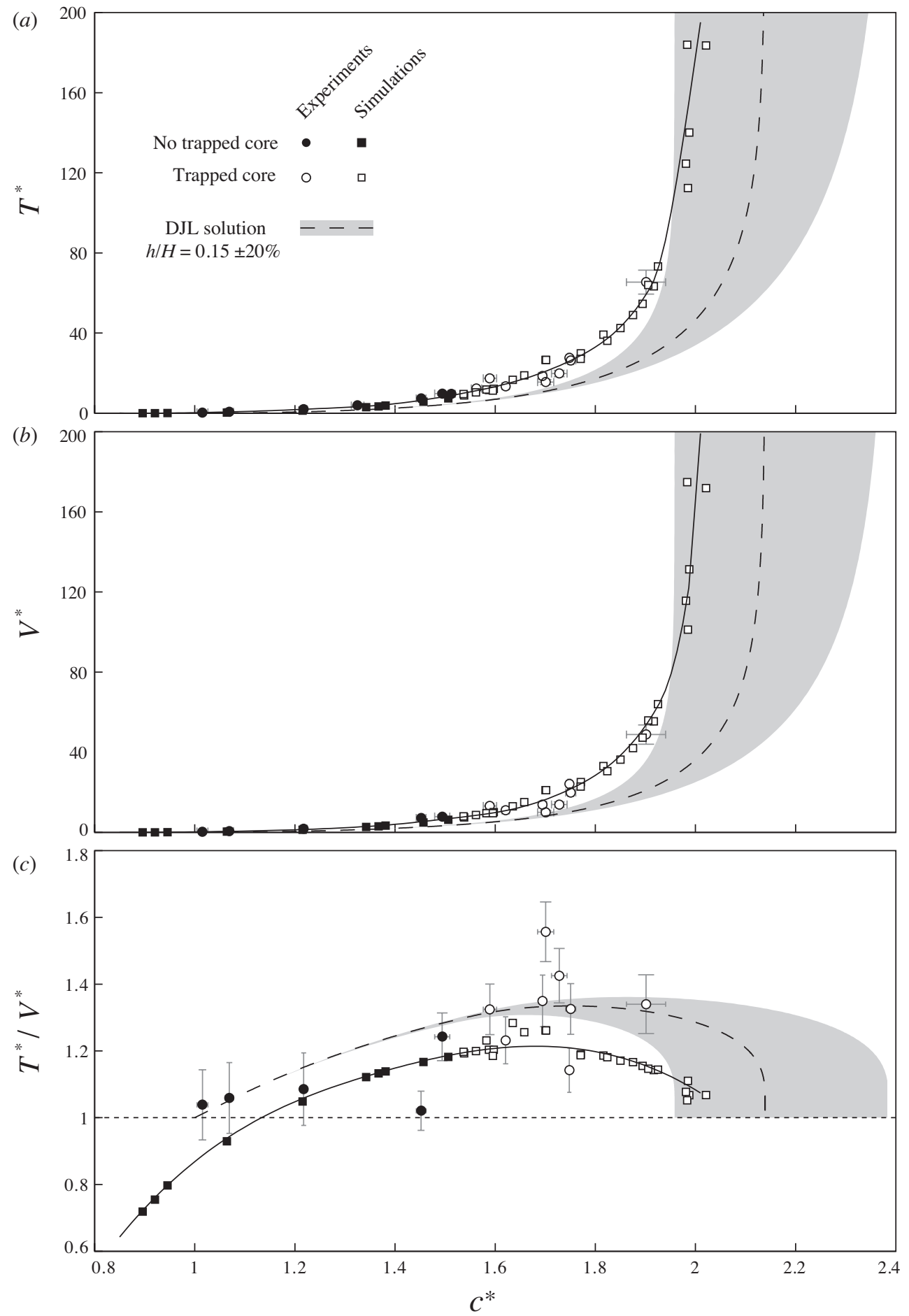

FiguRE 9. (a) kinetic energy, $(b)$ available potential energy and $(c)$ ratio of kinetic and potential energies. Symbols as in figure 5 . 


\section{Boundary layer effects in full-scale waves}

As noted in the previous section, while our results show good collapse, they exhibit a persistent offset from the DJL solutions, which we attribute to the presence of appreciably thick boundary layers in our experiments and simulations. For oceanic-scale waves, one might expect viscous effects to be negligible. Nevertheless, for solitary waves propagating along the bottom of the coastal ocean, or for atmospheric waves, a turbulent boundary layer does develop beneath the wave. It therefore seems interesting to try to estimate the thickness of these turbulent boundary layers, to assess whether in some instances they might be comparable to those encountered in our results.

We obtain a simple estimate of the boundary layer thickness by proceeding as follows. We assume that the vertical scale $\delta$ of the boundary layer may be approximated by the classic relation, for fully-developed flow over a flat plate:

$$
\frac{\delta}{x}=A R e_{x}^{-1 / n},
$$

where $x$ is a the downstream distance, $R e_{x}$ is the Reynolds number based on $x$, and $(A, n)$ are constants. For a laminar flow, $A=4.91, n=2$ (from the classical Blasius similarity solution), whereas for turbulent flow the empirical constants $A=0.382, n=5$ are typically used (Schlichting 1979). As noted above, this approximation assumes fully developed flow, which is typically not the case for long internal waves. Nevertheless, we might expect (5.1) to provide an estimate of the order of magnitude of $\delta / h$, if we set $x \sim \lambda$, and use $c$ for the characteristic velocity. Equation (5.1) can then be used to write $\delta / h$ in terms of the more appropriate Reynolds number $R e_{h}$ as:

$$
\frac{\delta}{h} \sim A\left(\frac{h}{\lambda}\right)^{-\frac{n-1}{n}} R e_{h}^{-1 / n},
$$

We can test this relation using our computational data. In figure 10, the continuous line shows a curve fit to our simulation results for the displacement thickness, whereas the dashed line is relation (5.2), evaluated using fits to the corresponding data for $\lambda, h$ and $c$, and $A$ and $n$ for laminar flows. While the trends are different, the results are within an $O(1)$ factor of each other, suggesting that (5.2) captures the order of magnitude of $\delta / h$, for this flow.

We next apply relation (5.2), using $(A, n)$ for turbulent flows, to observational data for bottom-propagating oceanic waves, using figures reported by Klymak \& Moum (2003); Scotti \& Pineda (2004) and Moum et al. (2007a). The resulting estimates of $\delta / h$ are shown by the open symbols in figure 10. Similarly, we also apply (5.2) to atmospheric solitary wave measurements from Clarke et al. (1981); Shreffler \& Binkowski (1981); Doviak \& Christie (1989) and Cheung \& Little (1990), shown by the closed symbols. While there is appreciable scatter, several values of $\delta / h$ are of the order of $10^{-1}$, and therefore comparable to our laboratory measurements. This data suggests that boundary-layer effects could possibly be appreciable also in some full-scale situations involving solitary internal waves of elevation.

\section{Testing empirical stability criteria}

Several empirical criteria for stability exist in the literature. In our experiments and simulations, a variety of unstable waves were observed. However, we are concerned about the source of these instabilities; more specifically, since the waves are produced using

a lock-release arrangement (as traditionally done also by previous studies), the waves emerge out of a vigorously unstable initial flow. Our aim, in this section, is therefore to 


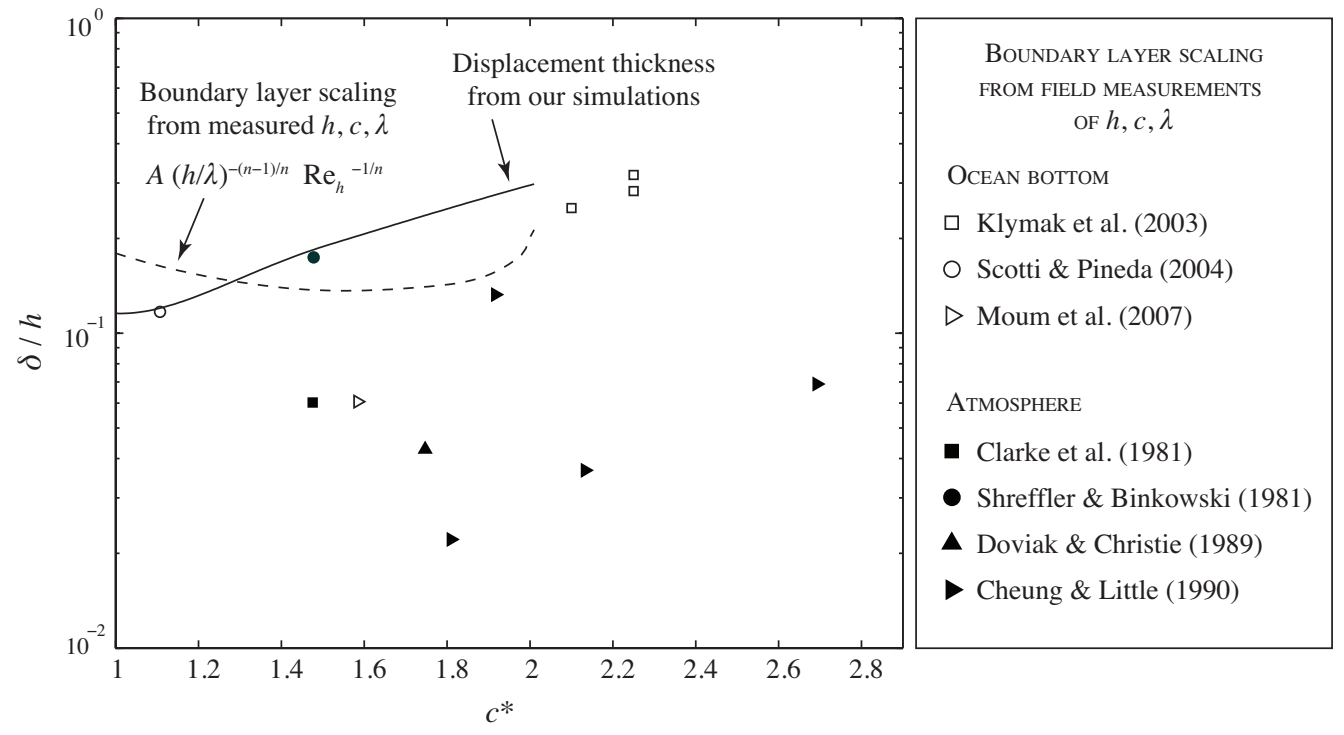

FiguRE 10. Order-of-magnitude estimate of nondimensional boundary layer thickness $\delta / h$, for the boundary layer adjacent to the stratified region. The continuous line shows a fit to our simulation results, whereas the dashed line shows an approximation obtained from classical theory for fully-developed flow. Symbols show estimates, from fully developed theory, for bottom-propagating ocean waves (open symbols) and atmospheric waves (closed symbols).

find the fastest waves that are stable, and test whether these might violate any of the available empirical stability criteria.

We used our experimental and numerical fields of velocity and density to compute fields of Richardson number $R i$, defined as

$$
R i=\frac{N^{2}}{\left(\frac{\partial u}{\partial z}\right)^{2}}
$$

We recorded its smallest value (that is, $\min (R i)$ ) occurring in the stratified region; if $\min (R i)<1 / 4$, we also recorded the extent of the surrounding region with $R i<1 / 4$. The interior of the core was excluded from these measurements, since we have established in the previous section that the core has essentially uniform density. We initially plotted data for $\left\langle\rho_{\tau}^{*}\right\rangle<0.06$ (as for the other plots shown so far); for the Richardson number, we observed considerable scatter in both the experimental and numerical results. For this reason, we further restricted the displayed data, in this section, to $\left\langle\rho_{\tau}^{*}\right\rangle<0.015$ (to help clarify what data meets this requirement, $\left\langle\rho_{\tau}^{*}\right\rangle$ for all data in previous plots is shown earlier in figure $7 c$ ). This resulted in a dataset whose size was sufficient to test in detail DJL predictions.

Figure 11 shows $\min (R i)$, as well as area, height and length for the $R i<1 / 4$ region. For $\min (R i)$, the DJL solution provides a reasonable approximation, especially after the onset of a trapped core. Similarly to other wave properties, the dimensions of the $R i<1 / 4$ region follow trends that are quite similar to those of the DJL solution, but display a nonuniform offset, which is likely due to viscous effects.

We use our results to test empirical stability criteria available in the literature. Troy \& Koseff (2005), in the course of experiments involving two uniform layers with a thin pycnocline, found that a $\min (R i)$ of around $0.07-0.08$ was necessary in order to obtain 
(a)

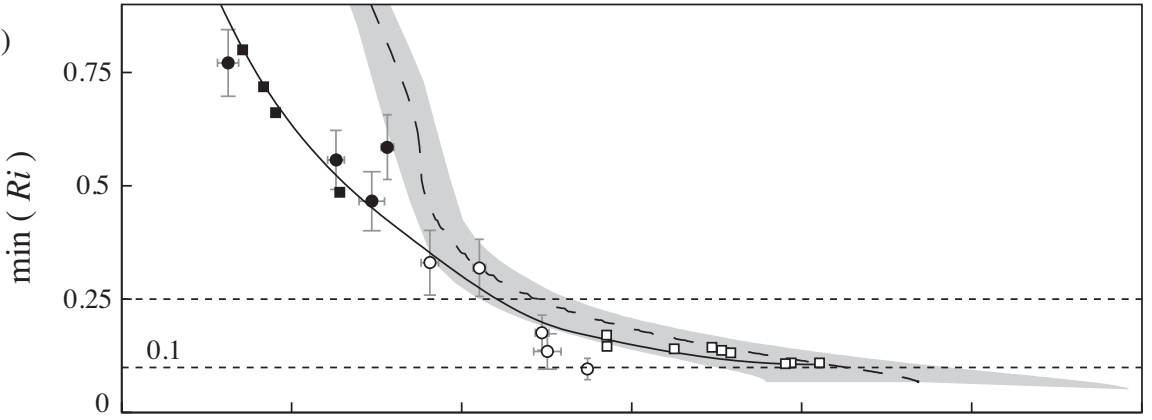

(b)

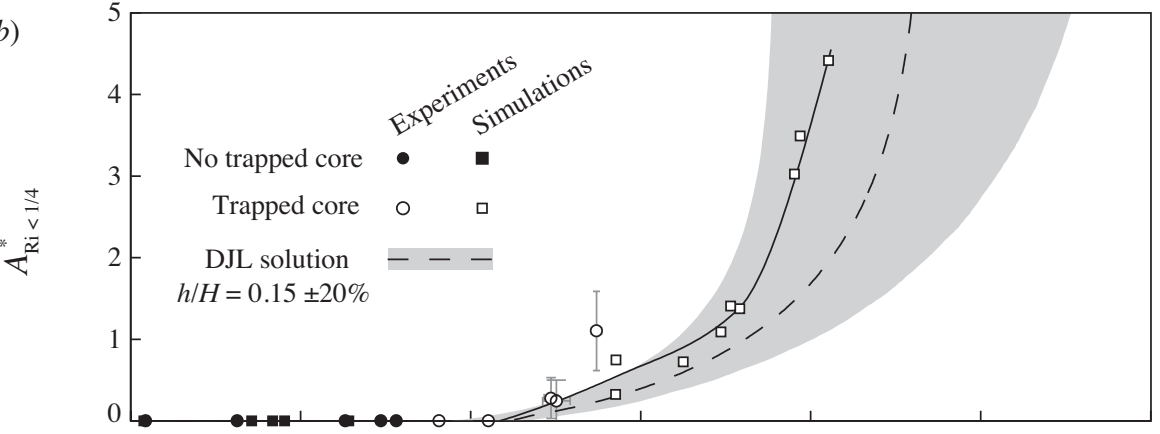

(c)

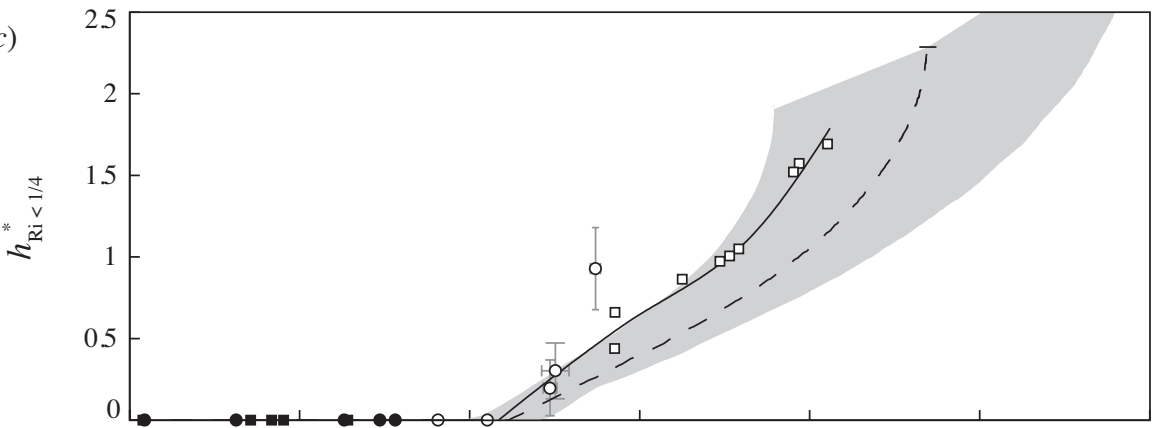

(d)

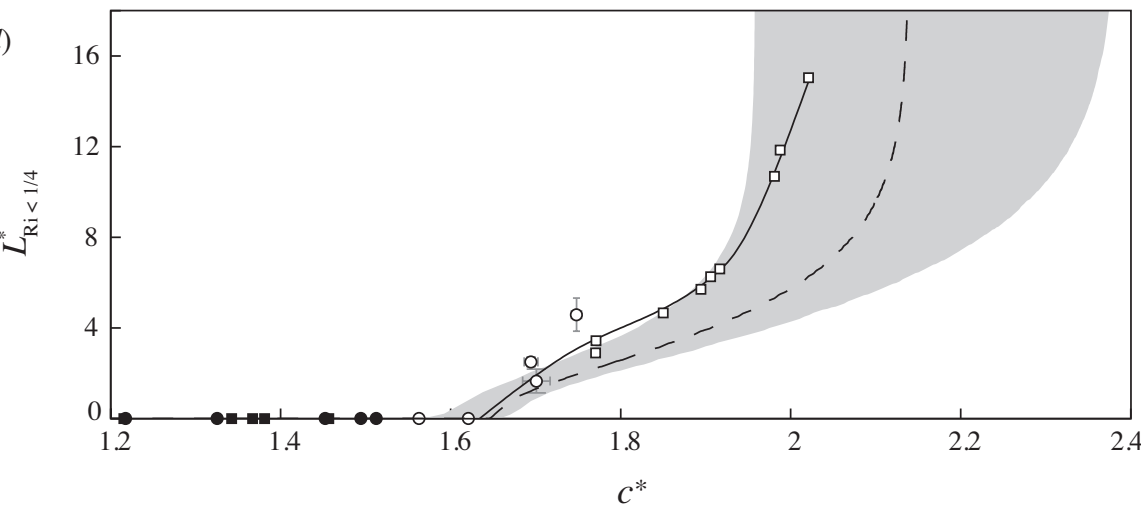

FiguRE 11. (a) minimum Richardson number versus phase velocity. The other panels show the geometric properties of the region with $R i<1 / 4$ outside of the trapped core. $(b),(c)$, and $(d)$ show the regionss area, height, and length, respectively. To ensure that these realizations are steady, the flows in this plot satisfy $\left\langle\rho_{\tau}^{*}\right\rangle<0.015$. 

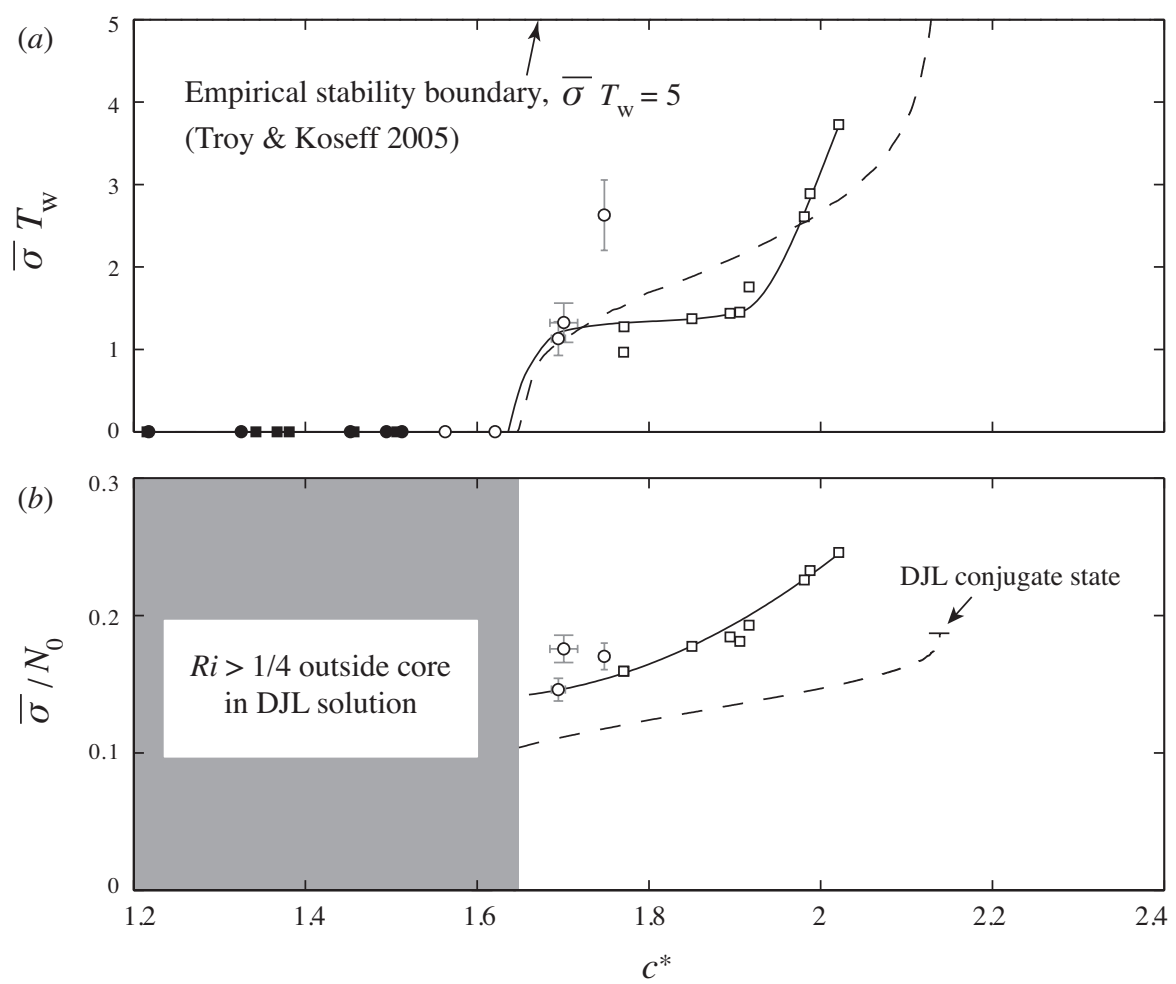

FiguRE 12. Test of the empirical stability boundary proposed by Troy \& Koseff (2005), requiring $\bar{\sigma} T_{w}<5$. This criterion was formulated on the basis of experimental results for waves without trapped cores. We find that this stability criterion also holds for our trapped-core waves. Symbols as in figure 11 .

overturning (through a Kelvin-Helmholtz instability). Recent numerical simulations by Barad \& Fringer (2010) found a similar threshold of $\min (R i)=0.1$. Figure 11(a) shows that (within experimental uncertainty) all our values of $\min (R i)$ are larger than 0.1 .

Troy \& Koseff (2005) also proposed a more refined stability criterion, based on an estimate of the fastest growth rate for the temporal stability problem. They used the Taylor-Goldstein equation for a parallel flow to perform a temporal stability analysis, and computed the fastest growth rate $\sigma$ at each $x$-location in the region $R i<1 / 4$. They found an empirical stability boundary as $\bar{\sigma} T_{w}=5$, where $\bar{\sigma}$ is the time-average of $\sigma$, and $T_{w}$ is the time that a fluid particle spends within the $R i<1 / 4$ region. Since the stability criterion of Troy \& Koseff (2005) was developed for interfacial waves, it is interesting to ask whether the same criterion holds for experimentally realized solicores.

We used the Taylor-Goldstein solver of Smyth et al. (2011) to perform a temporal stability analysis for our experiments and simulations (as well as for the DJL solutions). The resulting $\bar{\sigma} T_{w}$ is shown in figure 12(a). All of the waves in this figure are stable, and satisfy $\bar{\sigma} T_{w}<5$, consistently with the stability criterion of Troy \& Koseff (2005). For completeness, we also report $\bar{\sigma} / N_{0}$ in figure $12(b)$. In our experiments and simulations, we do not observe instabilities originating outside the core. Furthermore, instabilities originating inside the core appear only in the initial stages of wave propagation, suggesting that transient effects associated with the generation mechanism may be to blame (leading to a possible density mismatch between the core and ambient fluid; see $\S 4.1$ ). For this reason, we cannot test whether unstable waves meet the requirement $\bar{\sigma} T_{w}>5$. 


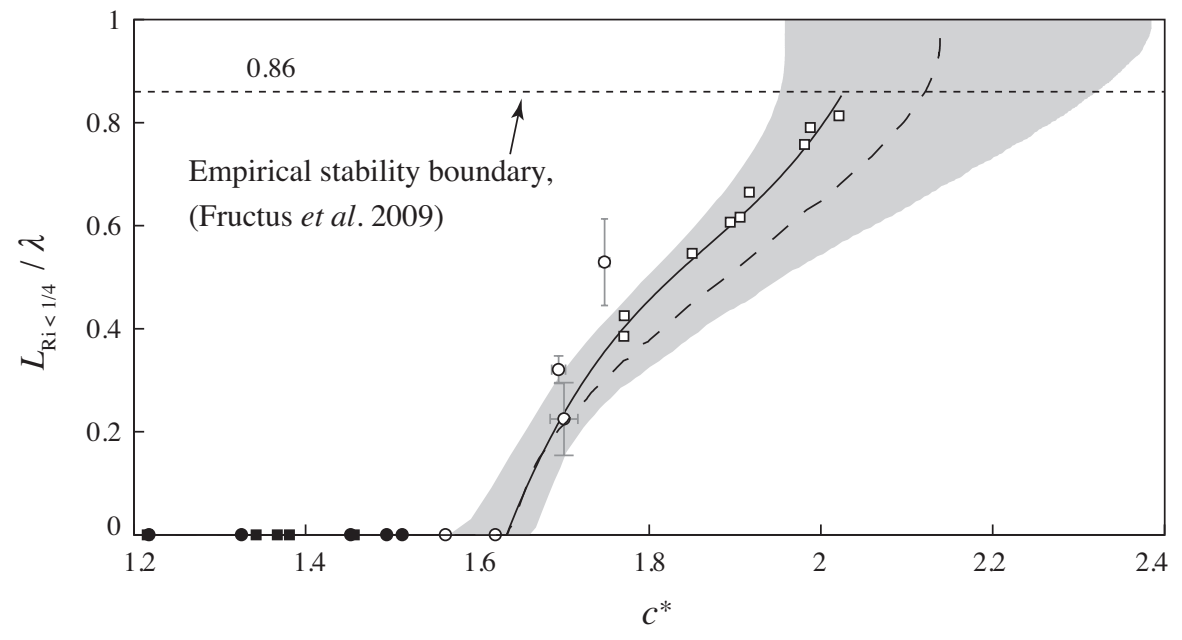

FIgURE 13. Test of the empirical stability boundary proposed by Fructus et al. (2009), requiring $L_{R i<1 / 4} / \lambda<0.86$, where $\lambda$ is the wavelength based on the half-amplitude. This criterion was formulated on the basis of experimental results for waves without trapped cores. We find that this stability criterion also holds for our trapped-core waves. Symbols as in figure 11.

Nevertheless, it is interesting to note that, as $c^{*}$ tends to its asymptotic value for large waves, $\bar{\sigma}$ does not grow particularly rapidly, whereas the increase in $T_{w}$ (corresponding to longer waves) accounts for most of the growth in $\bar{\sigma} T_{w}$. This suggests that it should be possible to encounter the $\bar{\sigma} T_{w}=5$ boundary, provided one can generate waves with sufficiently large wavelength.

It is worth briefly noting here that, while the calculation for $\bar{\sigma}$ is robust, we found that, for solicores, the value of $T_{w}$ is quite sensitive to the details of the calculation. This stems from the fact that the $R i<1 / 4$ region is typically contiguous to the core; therefore, at its upper boundary (near the core) the fluid velocity in the frame of reference moving with the wave is small, and increases rapidly with distance from the core. This is true also for the DJL solutions; for a DJL calculation with a vertical resolution of $\Delta z / H=6.25 \times 10^{-3}$, the calculated value of $T_{w}$ fluctuates by about $10 \%$ between neighboring solutions. The DJL results shown in figure 12(a) were filtered to remove these numerical fluctuations, being careful to preserve the location where $\bar{\sigma} T_{w}$ first becomes nonzero. (Due to the substantial computational cost, we also omitted the usual bounds on the DJL results in this figure.) Recently, Lamb \& Farmer (2011) formulated a similar stability criterion by solving the spatial stability problem, which offers more robust results for trapped-core waves. However, this is outside the scope of our current study.

Based on experimental results involving two homogenous layers separated by a sizable linearly stratified region, Fructus et al. (2009) proposed a simpler instability criterion, defined by the threshold $L_{R i<1 / 4} / \lambda=0.86$. Here, $L_{R i<1 / 4}$ is the length of the region outside the core with $R i<1 / 4$, whereas $\lambda$ is the wavelength based on the wave's halfamplitude (that is, $\lambda$ is the horizontal extent of the region where the magnitude of the interface displacement is at least half of its largest value). A plot of $L_{R i<1 / 4} / \lambda$ is reported in figure $13(a)$. Our results, corresponding to stable waves, are all below 0.86 , consistently with the criterion of Fructus et al. (2009).

Finally, we should briefly note that, for similar values of $h / H$ and $c^{*}$, Carr et al. (2008) found instabilities of varying degree (as summarized by their table 1), whereas all our results show stable waves. The reason for the discrepancy is not immediately clear. We 
hypothesize that this difference in stability properties might be attributable to some unsteady effects dependent on the initial conditions. This dependence would have been substantially mitigated in our results, through the introduction of the steadiness criterion embodied by (4.1).

\section{Conclusions}

In this paper, we employed a recently devised experimental technique, enabling simultaneous, co-planar measurements of velocity and density using pulsed lasers, and investigated the structure and stability of solitary internal waves with trapped cores (for brevity, we refer to these flows as "solicores").

We first examined waves propagating near a free surface; in this case, all solicores exhibited a strong shear instability. We proposed that Marangoni effects are to blame for this instability. These effects arise as a consequence of a strong convergent surface flow associated with the front of the trapped core, thereby locally accumulating surfactant, leading to strong surface-tension-driven shear on the surface, ahead of the wave. When this shear layer interacts with the core, it is subducted into the fluid interior, becoming susceptible to Kelvin-Helmholtz instability. We used our velocity data to perform quantitative calculations that support this hypothesis.

This deduction led us to performing experiments with a no-slip lid, where we observed robust waves with large cores. For very large amplitudes, we found it useful to complement our results with viscous numerical simulations, employing a longer virtual tank, which allowed initial transient effects to dissipate.

We proposed computing the core region as a Lagrangian coherent structure (instead of using a closed streamline, as had traditionally been done in the literature), as this enables a more robust core definition. In addition, we introduced a steadiness criterion, based on the measured core dynamics, and used this condition to select within our experiments a family of quasi-steady, robust flows, which exhibits good collapse in wave properties.

Our simultaneous velocity and density data enabled us to characterize in detail the core properties. The core circulation is small (at most, around $10 \%$ of the baroclinic wave circulation) and is therefore expected to be dynamically unimportant. The core density is essentially uniform (with the density standard deviation in the core region being less than $4 \%$ of the full density range). This constitutes a key finding of this study, and indicates that models with irrotational, uniform-density cores should be used to study these waves.

We reported in detail the amplitude, circulation, energetics and core geometric properties of these waves. We find that these results are consistent with predictions from Dubreil-Jacotin-Long (DJL) theory, except for an offset that is likely due to viscous effects.

Finally, we used our results to test the empirical stability criteria of Troy \& Koseff (2005) and of Fructus et al. (2009). To this end, we computed fields of Richardson number, and performed a temporal stability analysis based on a parallel-flow approximation near the wave trough, using the Taylor-Goldstein equation. We found that all of our stable waves are indeed on the "stable" side of each instability threshold, indicating that our measurements for solicores are consistent with these stability criteria.

A number of interesting directions remain to be pursued. As noted earlier in this section, DJL predictions and experimental results, while consistent with each other, show a perceivable offset, which is likely due to finite-Re effects. It would be of interest to repeat some of these experiments at a larger-scale facility, such as the one described by Grue et al. (2000). It would also be valuable to develop a refinement of DJL theory that would 
account for this offset, as this would enable a precise comparison between DJL theory and laboratory data, even for smaller-scale facilities. Furthermore, it would be interesting to perform a linear stability analysis of the complete, two-dimensional flow, as this would reveal the detailed structure of each instability that can arise in these waves. We hope to report our progress on some of these questions in a future paper.

Funding from NSF grant OCE-1029672 is gratefully acknowledged. P.L.F. is thankful for support from the Postdoctoral Scholar program at the Woods Hole Oceanographic Institution, and for funding from the Devonshire Foundation. The preparation of this manuscript was supported by a Junior Research Fellowship from Churchill College, Cambridge. We are grateful to Anders Jensen for his work on the construction of the wave tank, and for his assistance with running the experiments.

\section{REFERENCES}

Akylas, T. R, Grimshaw, R. H. J., Clarke, S. R. \& Tabaei, A. 2007 Reflective tidal internal wave beams and local generation of solitary internal waves in the ocean thermocline. $J$. Fluid Mech. 593, 297-313.

Almgren, A. S., Bell, J. B., Colella, P., Howell, L. H. \& Welcome, M. L. 1998 A conservative adaptive projection method for the variable density incompressible NavierStokes equations. J. Comp. Phys. 142 (1), 1-46.

Barad, M. F. \& Fringer, O. B. 2010 Simulations of shear instabilities in interfacial gravity waves. J. Fluid Mech. 644, 61-95.

BAtchelor, G. K. 1956 On steady laminar flow with closed streamlines at large Reynolds number. J. Fluid Mech. 1, 177-68.

Carr, M., Fructus, D., Grue, J., Jensen, A. \& Davies, P. A. 2008 Convectively induced shear instability in large amplitude internal solitary waves. Physics of Fluids 20 (12), 126601.

Cheung, T. K. \& Little, C. G. 1990 Meteorological tower, microbarograph array, and sodar observations of solitary-like waves in the nocturnal boundary layer. J. Atmos. Sci. 47, $2516-2536$.

Clarke, R. H., Smith, R. K. \& Reid, D. G. 1981 The morning glory of the Gulf of Carpentaria: an atmospheric undular bore. Monthly Weather Review 109 (8), 1726-1750.

DAvis, R. E. \& Acrivos, A. 1967 Solitary Internal Waves in Deep Water. J. Fluid Mech. 29, 593-607.

Derzho, O. G. \& Grimshaw, R. H. J. 1997 Solitary waves with a vortex core in a shallow layer of stratified fluid. Phys. Fluids 9 (11), 3378-3385.

Diamessis, P. J. \& Redekopp, L. G. 2006 Numerical investigation of solitary internal waveinduced global instability in shallow water benthic boundary layers. J. Phys. Ocean. 36 (5), 784-812.

Doviak, R. J. \& Christie, D. R. 1989 Thunderstorm-generated solitary waves - A wind shear hazard. J. Aircraft 26 (5), 423-431.

Dubreil-Jacotin, M. L. 1934 Sur la détermination rigoureuse des ondes permanentes périodiques d'ampleur finie. J. Math. Pure Appl. 13, 217291.

Economidou, M. \& Hunt, G. R. 2008 Density stratified environments: the double-tank method. Exp. Fluids 46 (3), 453-466.

Fructus, D., Carr, M., Grue, J., Jensen, A. \& A., Davies P. 2009 Shear-induced breaking of large internal solitary waves. J. Fluid Mech. 620, 1-29.

Grimshaw, R. H. J. 1969 On steady recirculating flows. J. Fluid Mech. .

Grue, J., Jensen, A., Rusas, P. O. \& Sveen, J. K. 2000 Breaking and broadening of internal solitary waves. J. Fluid Mech. 413, 181-217.

HAller, G. 2005 An objective definition of a vortex. J. Fluid Mech. 525, 1-26.

HEAD, M. J. 1983 The use of miniature four-electrode conductivity probes for high resolution measurement of turbulent density or temperature variations in salt-stratified water flows. PhD thesis, UC San Diego. 
Helfrich, K. R. \& White, B. L. 2010 A model for large-amplitude internal solitary waves with trapped cores. Nonlin. Processes Geophys. 17, 303-318.

King, S. E., Carr, M. \& Dritschel, D. G. 2011 The steady-state form of large-amplitude internal solitary waves. J. Fluid Mech. 666, 477-505.

Klymak, J. M. \& Moum, J. N. 2003 Internal solitary waves of elevation advancing on a shoaling shelf. Geophys. Res. Lett. 30 (20), 2045.

Kropfli, R. A., Ostrovski, L. A., Stanton, T. P., Skirta, E. A., Keane, A. N. \& Irisov, V. 1999 Relationships between strong internal waves in the coastal zone and their radar and radiometric signatures. J. Geophys. Res. 104, 3133-3148.

LAmB, K. G. 2002 A numerical investigation of solitary internal waves with trapped cores formed via shoaling. J. Fluid Mech. 451.

LAmb, K. G. \& FARMER, D. 2011 Instabilities in an Internal Solitary-like Wave on the Oregon Shelf. J. Phys. Ocean. 41 (1), 67-87.

Lamb, K. G \& NGuyen, V. T. 2009 Calculating Energy Flux in Internal Solitary Waves with an Application to Reflectance. J. Phys. Ocean. 39 (3), 559-580.

LaW, A. W. K. \& WANG, H. W. 2000 Measurement of mixing processes with combined digital particle image velocimetry and planar laser induced fluorescence. Exp. Thermal Fluid Sci. 22, 213-229.

LeAl, L. G. 2007 Advanced Transport Phenomena: Fluid Mechanics and Convective Transport Processes (Cambridge Series in Chemical Engineering). Cambridge University Press.

Lien, R. C., D'Asaro, E. A., Henyey, F., Chang, M., Tang, T. \& Yang, Y. 2012 Trapped core formation within a shoaling nonlinear internal wave. J. Phys. Ocean. 42 (4), 511-525.

LoNG, R 1953 Some aspects of the flow of stratified fluids: I. A theoretical investigation. Tellus 5 (1), 42-58.

Melton, L. A. \& Lipp, C. W. 2003 Criteria for quantitative PLIF experiments using highpower lasers. Exp. Fluids 35 (4), 310-316.

Mercier, M. J., Martinand, D., Mathur, M., Gostiaux, L.and Peacock, T. \& Dauxois, T. 2010 New wave generation. J. Fluid Mech. 657, 308-334.

Moum, J. N., FArmer, D. M., Shroyer, E. L., Smyth, W. D. \& Armi, L. 2007 a Dissipative losses in nonlinear internal waves propagating across the continental shelf. J. Phys. Ocean. 37 (7), 1989-1995.

Moum, J N, Klymak, J M, Nash, J D, Perlin, A \& Smyth, W D $2007 b$ Energy Transport by Nonlinear Internal Waves. J. Phys. Ocean. 37 (7), 1968-1988.

Rao, M. P., Castracane, P., Casadio, S., Fuá, D. \& Fiocco, G. 2004 Observations of atmospheric solitary waves in the urban boundary layer. Boundary-Layer Meteorology 111 (1), 85-108.

Rosen, M. J. \& Kunjappu, J. T. 2012 Surfactants and interfacial phenomena. Wiley.

Salloum, M., Knio, O. M. \& Brandt, A. 2012 Numerical simulation of mass transport in internal solitary waves. Phys. Fluids 24 (1), 016602.

Schlichting, H. 1979 Boundary layer theory. McGraw Hill.

Scotti, A. \& PinedA, J. 2004 Observation of very large and steep internal waves of elevation near the Massachusetts coast. Geophys. Res. Lett. 31 (22), 22307.

Shadden, S. C., Dabiri, J. O. \& Marsden, J. E. 2006 Lagrangian analysis of fluid transport in empirical vortex ring flows. Phys. Fluids .

Shreffler, J. H. \& Binkowski, F. S. 1981 Observations of pressure jump lines in the Midwest, 10-12 August 1976. Mon. Weather Rev. 109 (8), 1713-1725.

Sмyтh, W. D., Moum, J. N. \& Nash, J. D. 2011 Narrowband oscillations in the upper equatorial ocean. Part II: properties of shear instabilities. J. Phys. Ocean. 41 (3), 412-428.

Sutherland, B. R., Barrett, K. J. \& Ivey, G. N. 2013 Shoaling internal solitary waves. J. Geophys. Res.-Oceans 118 (9), 4111-4124.

Troy, C. D. \& Koseff, J. R. 2005 The instability and breaking of long internal waves. J. Fluid Mech. 543, 107-136.

Wu, M. \& Gharib, M. 2002 Experimental studies on the shape and path of small air bubbles rising in clean water. Phys. Fluids 14 (7), L49. 